Dear author,

Please note that changes made in the online proofing system will be added to the article before publication but are not reflected in this PDF.

We also ask that this file not be used for submitting corrections. 


\title{
Studying model suspensions using high resolution synchrotron X-ray microtomography
}

\author{
${ }_{3}$ Q1 Syed F. Islam ${ }^{a, *}$, Lucia Mancini ${ }^{b}$, Ramana V. Sundara ${ }^{c}$, Steve Whitehouse ${ }^{c}$, \\ Stefan Palzer ${ }^{d}$, Michael J. Hounslow ${ }^{a}$, Agba D. Salman ${ }^{a}$ \\ a Department of Chemical and Biological Engineering, University of Sheffield, Sheffield S1 3JD, UK \\ 6 Q2 b Elettra-Sincrotrone Trieste S.C.p.A., S.S. 14-km 163.5 in Area Science Park, Trieste, 34149 Basovizza, Italy \\ c Nestlé Product Technology Centre, Haxby Road, York YO31 8TA, UK \\ d Nestlé SA Headquarters, Avenue Nestlé 55, CH-1800 Vevey, Switzerland
}

\section{A R T I C L E I N F O}

\section{Article history:}

Received 19 August 2016

Received in revised form 24

November 2016

Accepted 28 November 2016

Available online $\mathrm{xxx}$

\section{Keywords:}

Synchrotron X-ray

microtomography

X-ray computed tomography

Dynamic $\mu \mathrm{CT}$

Suspensions

Sucrose

${ }_{26}$ Q4 Secondary immiscible liquid Sunflower oil

\begin{abstract}
A B S T R A C T
The addition of minor quantities of secondary liquids to suspensions may lead to a transition from a fluid-like structure to paste-like structure for the system. Previous studies have shown how rheological properties such as viscosity and yield stress are affected, however, qualitative visual observation on the micro-scale during both short and long term storage has yet to be achieved or reported.

This research focuses on the movement of a secondary immiscible liquid (water or saturated sucrose solution) when added to a model food system. The model food system used in this study is a suspension of sucrose particles in a continuous oil phase to better understand the interactions between the particles and the liquid phases present. This was accomplished using dynamic X-ray computer tomography to study the behaviour of the sample. This nondestructive approach allowed the movement of the secondary liquid as well as the solid particles from the bulk suspension to be monitored through a time lapse of scans. This was achieved by observing the changes in the grey scale range of the droplet with time, which was then correlated to the uptake and movement of sucrose into the secondary liquid using an innovative method. This movement was due to the hydrophilicity and solubility of sucrose with gravity/sedimentation playing a minimal role.
\end{abstract}

(c) 2016 The Author(s). Published by Elsevier B.V. on behalf of Institution of Chemical Engineers. This is an open access article under the CC BY license (http://creativecommons. org/licenses/by/4.0/).

\section{Introduction}

28Q5 Several food products comprise of suspensions in which solid hydrophilic particles are suspended as a disperse phase throughout a continuous hydrophobic liquid phase. When a small proportion of a secondary immiscible liquid such as water or glycerol is added to a suspension of this kind, it can lead to behavioural changes from a fluid-like material to a paste-like material (Koos and Willenbacher, 2011; Johansson and Bergenståhl, 1992). This transformation has been ascribed to the formation of liquid bridges between the solid particles by the secondary liquid thereby modifying the rheological properties of the suspension such as its yield stress and viscosity which can increase several-fold (Koos and Willenbacher, 2011, 2012; Koos et al.,

Q3 * Corresponding author.

E-mail address: sfislam1@sheffield.ac.uk (S.F. Islam). http://dx.doi.org/10.1016/j.cherd.2016.11.034

0263-8762/@ 2016 The Author(s). Published by Elsevier B.V. on behalf of Institution of Chemical Engineers. This is an open access article under the CC BY license (http://creativecommons.org/licenses/by/4.0/).
2012; Negreiros et al., 2015). The secondary liquid in this case has a greater affinity to the primary particles in comparison to the continuous phase because of their hydrophilic nature.

In food systems, the movement of moisture is generally considered from the view point of molecular diffusion. Moisture can enter from the surrounding atmosphere and/or may diffuse between different regions within the product. This movement is driven by the water activity differential between the different domains. This is of particular importance when considering the preservation of food products and the behaviour changes which may occur during storage. Past studies in the literature have focused on the assumption of diffusion being the dominant mechanism for moisture movement within such systems, and models are presented based on apparent or effective diffusivities to simplify the kinetics taking place (Yuan et al., 2009, 2012). These studies focused on the bulk mass transfer as an overall average of the sample, but did not investigate the localised movement. A more plausible explanation would be that a combination of transport 
mechanisms are responsible for the movement of a secondary liquid phase in a suspension including gravity, capillary and diffusion effects (Ghosh et al., 2004). However, at present, there is limited work within the literature that has been able to follow the localised movement of a secondary immiscible liquid within a suspension and qualitatively track the interactions and structural changes taking place.

$\mathrm{X}$-ray computed microtomography $(\mu \mathrm{CT}$ ) has been used previously to characterise several properties of food products in three dimensions (3D) such as the cellular structure of cereal bars (Chevallier et al., 2014; Falcone et al., 2004), analyse bread, biscuits and breadsticks (Frisullo et al., 2010a; Falcone et al., 2006), coffee beans (Pittia et al., 2011), the microstructure of apples stored at various environmental conditions (Herremans et al., 2014), the microstructure of mayonnaise (Laverse et al., 2012) and of various chocolates (Frisullo et al., 2010b; Reinke et al., 2015) to name a few.

The current work uses a state-of-the-art non-invasive method based on phase-contrast synchrotron X-ray $\mu \mathrm{CT}$ to monitor the movement of a secondary liquid within a suspension through a series of scans taken at different time intervals (dynamic or 4D $\mu \mathrm{CT}$ ). Using this method it was possible to track behavioural effects over timescales of interest for food stability studies.

\section{Materials and methods}

\subsection{Materials}

Sucrose suspensions (icing sugar, ZMR Zuckermühle Rupperswil AG, Germany) and high oleic sunflower oil (Surface tension $35.5 \mathrm{mN} / \mathrm{m}$, FTA125. Viscosity $49 \mathrm{mPa}$, Malvern Kinexus lab+. Measured at $25^{\circ} \mathrm{C}$ ) were prepared according to the method described by Islam et al. (2016). The mass fractions of the solid and liquid phases were 50\% wt for both; this amount was chosen based on the particle size distribution of the sucrose; ensuring adequate sunflower oil is present to completely coat all particles. The solid particles had a size distribution of $9-110 \mu \mathrm{m}$, with a median particle size $\left(d_{50}\right)$ of $32 \mu \mathrm{m}$ (measured using Malvern Mastersizer S, Malvern Instruments, UK).

Once the suspensions were prepared, they were mixed manually with a glass rod for a further $30 \mathrm{~s}$ and then transferred into cellulose straws ( $9 \mathrm{~mm}$ diameter, Fishers Ideas UK) which were glued (to prevent leaking of sample) to a plastic chuck specifically designed for the X-ray $\mu$ CT experiments using a disposable pipette. A specifically prepared lid with a hole in the centre to fit the pipette tip was then placed on top, which ensured that the addition of the secondary liquid was to the centre of the suspension. The secondary liquid, which was either distilled water or a saturated sucrose solution, was added to the surface of the suspension using an electronic pipette (Eppendorf Xplorer, UK), locating the tip of the pipette close to the surface of the suspension, though not actually touching the surface. The distance between the surface of the suspension and the pipette tip was approximately $5 \mathrm{~mm}$. Once the secondary liquid was added, another lid was placed which contained no holes, to prevent the evaporation of the secondary liquid during the experiment.

\subsection{Rheological properties}

The rheological properties were measured using a rotational rheometer (Kinexus Lab+, Malvern, UK). For liquid samples a cone \& plate geometry was used $\left(1 / 50\right.$, with a $1^{\circ}$ angle and $50 \mathrm{~mm}$ diameter). Measurements were conducted using a shear rate ramp between the range of $10-1000 \mathrm{~s}^{-1}$, ensuring steady state had been reached at each step before proceeding to the next set shear rate.

For the suspension sample, a serrated parallel geometry was used (diameter of upper and lower plate of $40 \mathrm{~mm}, 1 \mathrm{~mm}$ gap). The serrated plate geometry was used to prevent slippage of the sample, as the bulk solid can settle creating layer oil on the surface. Measurements were conducted using a shear rate ramp between the range of $0.1-100 \mathrm{~s}^{-1}$, ensuring steady state had been reached at each step before proceeding to the next set shear rate. The data was fitted to a Herchel-Bulkley model to calculate the yield stress using Eq. (1):

$\sigma=\sigma_{y}^{H}+k \dot{\gamma}^{n}$

where $\sigma$ is the shear stress $(\mathrm{Pa}), \sigma_{y}^{H}$ is the yield stress $(\mathrm{Pa}), \dot{\gamma}$ is the shear rate $\left(\mathrm{s}^{-1}\right)$ with $k$ and $n$ are constants (Mewis and Wagner, 2012). All experiments were conducted at a temperature of $25^{\circ} \mathrm{C}$

\subsection{Scanning Electron Microscope (SEM)}

Images were taken using a Scanning Electron Microscope (SEM) (JEOL, JSM-6010LA) to visually observe both primary particles, as well as, the mixture of water/sucrose droplet after removal from the sucrose and sunflower oil suspension.

Images were taken to visualise the surface topography of the sample after an initial gold coating which is needed for obtaining an image after applying a focused beam of electrons. Scans were conducted at a moderate energy level of $20 \mathrm{kV}$, which was found to be appropriate after trialling various energy levels for the samples being scanned.

\subsection{Synchrotron X-ray $\mu \mathrm{CT}$ measurements}

Transmission X-ray $\mu \mathrm{CT}$ is a non-destructive technique based on the mapping of the linear attenuation coefficient of X-rays crossing the studied sample (Kak and Slaney, 1988) and allowing the internal 3D microstructure of a sample to be visualised and analysed. This is achieved by capturing a series of projections, as the sample is rotated between the X-ray source and detector. These projections are then reconstructed using an algorithm which allows the 3D internal structure at the micron-scale spatial resolution to be seen.

All samples studied in this work have been imaged at the SYRMEP beamline of the Elettra-Sincrotrone Trieste laboratory (Basovizza, Italy). A schematic view of the employed experimental setup can be seen in Fig. 1.

Performing synchrotron X-ray $\mu \mathrm{CT}$ experiments at a third-generation synchrotron source, such as Elettra, highly improves the image quality compared to the use of conventional X-ray sources. In fact, a synchrotron X-ray beam features properties that significantly increase the data quality, in terms of contrast and spatial resolution, and then extend the imaging possibilities. These properties are the beam monochromaticity, its high intensity, high spatial coher-

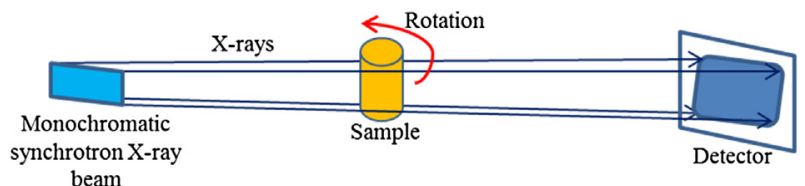

Fig. 1 - Schematic view of the experimental set up used for $\mathrm{X}$-ray $\mu \mathrm{CT}$ at the SYRMEP beamline of Elettra. 
ence and nearly-parallel geometry. A monochromatic and highly-intense X-ray beam leads to reconstructed slices free of beam hardening effects (Baruchel et al., 2000) and with a high signal-to-noise ratio.

Moreover, the high transverse coherence length characterising the X-ray beam, allows to apply phase-contrast imaging techniques. In phase-contrast $\mathrm{X}$-ray imaging the final contrast in the recorded image will be a superposition of absorption contrast (related to the imaginary part, $\beta$, of the refraction index of the material under study) with a contrast related to the refraction of the X-ray beam (interference between parts of the wavefront which experienced different phase shifts within the sample). Phase shifts are associated with sample features inducing phase variations in the X-ray beam and will be related to the non-imaginary part, $\delta$, of the refraction index (Cloetens et al., 1996).

The result is that in phase-contrast imaging, all the interfaces corresponding to abrupt phase changes in the sample show an enhanced visibility thanks to the presence of Fresnel fringes. This technique is particularly powerful for the visualisation of light materials or materials with small differences in refraction indices which would be hardly visible or sometime indistinguishable in absorption mode.

In the case of a nearly-parallel X-ray beam, it is possible to detect phase-contrast effects in the sample projections (radiography) or in the reconstructed slices $(\mu \mathrm{CT})$ by using an experimental set up simply based on the free-space propagation of the transmitted X-ray beam between the sample and the detector (Cloetens et al., 1996). In this approach, a crucial parameter is represented by the sample-to-detector distance $d$. Working in the 'near-field' regime, objects will appear sharper than in absorption mode, but to be able to extract reliable morphological parameters from the reconstructed $\mu \mathrm{CT}$ images phase-contrast artefacts are a significant problem and it is necessary to apply a 'filtering procedure' also called phase retrieval. This procedure will also help to increase the density contrast between materials with close mass density and will permit a good segmentation and separation of these materials.

In this work we used a single distance, phase retrieval algorithm proposed by Paganin et al. (2002). The consequence of this processing is generally a slight blurring of images, since acquiring conditions can be quite far from the ideal ones ( homogeneous monophase "phase objects" and perfectly monochromatic X-ray beam). These algorithms can be employed, with some caution, even on dense materials and with polychromatic light (Myers et al., 2007).

To apply the Paganin's algorithm it is needed to compute the ratio $(\gamma=\delta \beta)$ at the wavelength $(\lambda)$ used in the experiment. Practically, it is needed to tune the $\gamma$ ratio in order to maximise the filtering effect keeping at minimum the image blurring produced by the filtering procedure itself (Paganin et al., 2002).

Suspensions were positioned in cellulose straws (Fishers Ideas, UK). The beamline monochromator, based on a doubleSi (111) crystal system working in Bragg configuration, was set in order to tune the X-ray energy at $17 \mathrm{keV}$ (energy resolving power on the order of $10^{-4}$ ). For each sample 900 projections were acquired with equiangular steps over a range of $180^{\circ}$, with a regular angular step of $0.2^{\circ}$. The projections were recorded with a 12 bit, water-cooled, Photonic Science XDI-VHR CCD camera, consisting of a full frame CCD imager coupled to a gadolinium oxy sulphide scintillator by a fibreoptic taper. The camera has a $4008 \times 2672$ pixel chip. The effective pixel size of the detector is $4.5 \mu \mathrm{m}$. In our exper- iments, the pixel size has been fixed to $9.0 \mu \mathrm{m}$, applying a $2 \times 2$ binning, thus yielding a field of view of $18 \mathrm{~mm}$ (horizontal) $\times 12 \mathrm{~mm}$ (vertical). The total scan time for each sample was $24 \mathrm{~min}$. For all the investigated specimens, the distance $d$ was set to $150 \mathrm{~mm}$.

\subsection{Image analysis}

Scans were conducted on suspensions of sucrose and sunflower oil ( $50 \%$ wt for both). Once prepared, the addition of a fixed volume of secondary liquid $(5 \mu l$ or $10 \mu l$ water or saturated sucrose solution) was applied to the suspension without letting the suspension settle. Scans were conducted frequently initially after the secondary liquid addition, and then the time between scans was prolonged as the time from initial addition increased. Each scan took approximately $24 \mathrm{~min}$. During this period of $24 \mathrm{~min}$, the secondary liquid is moving and this needs to be taken into account when analysing the results.

All the acquired data sets were phase-retrieved and then reconstructed using the custom developed software SYRMEP Tomo Project (Brun et al., 2015) using the filtered back projection reconstruction algorithm (Kak and Slaney, 1988). The optimized $\gamma$ value for phase retrieval was set to 400 . The slices were stacked to obtain volumes where the isotropic voxel has an edge size of $9.0 \mu \mathrm{m}$. All reconstructed 3D datasets contained 801 slices.

Reconstructed slices from the X-ray scans were processed using the software Image J. The images were converted to 16 bit images (grey range intensity of $0-65,535$, where 0 corresponds to the darkest possible shade being black and 65,535 corresponds to the lightest possible shade being white and in between are shades of grey), which were then analysed to track the change in grey value of the droplet with time from the sequence of scans. The slice chosen for the analysis corresponded to when the secondary liquid is largest in terms of diameter along the $\mathrm{x}$-axis. A schematic for the axis being mentioned can be seen in Fig. 2.

All 2D slices shown for the different volumes and type of secondary liquid added to the suspension were opened in an image stack using ImageJ. For the stack, the maximum interval of grey values was adjusted to make the regions of interest more visible and this was then applied to the complete stack of images. Then the stacks of images were saved as individual tiff files, to which the analysis of the grey value was carried out. This ensured all images were adjusted using the same grey values interval, and the same adjustment was made for all scans of a given sample.

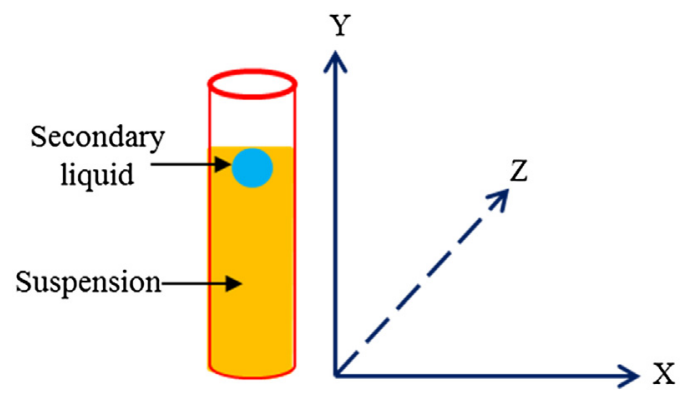

Fig. 2 - Representation of axis in relation to the sample and reconstructed scan slices. 


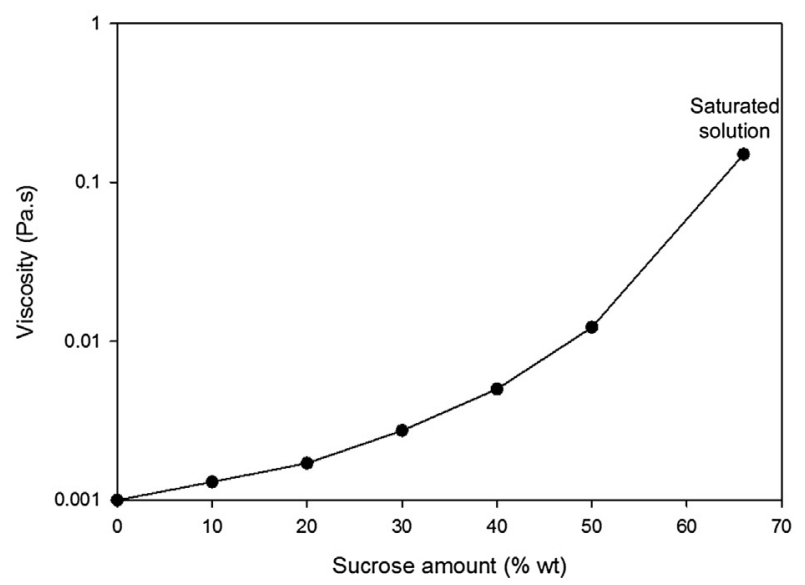

Fig. 3 - Viscosity measurements for different concentration sucrose solutions (from distilled water $(0 \% \mathrm{wt})$ to saturated sucrose solution $(67 \% \mathrm{wt})$ at $25^{\circ} \mathrm{C}$, average value for shear rate range between $\left.100-1000 \mathrm{~s}^{-1}\right)$.

Table 1 - Viscosity and density values for water and sucrose solutions.

\begin{tabular}{lll} 
& Viscosity $(\mathrm{mPa})$ & Density $\left(\mathrm{kg} / \mathrm{m}^{3}\right)^{\mathrm{a}}$ \\
\hline Distilled water & 0.98 & 1000 \\
$10 \%$ wt suc solution & 1.30 & 1040 \\
$20 \%$ wt suc solution & 1.71 & 1083 \\
$30 \%$ wt suc solution & 2.74 & 1129 \\
$40 \%$ wt suc solution & 5.00 & 1179 \\
$50 \%$ wt suc solution & 12.2 & 1232 \\
Saturated suc solution & 150 & 1337
\end{tabular}

a Values from Asadi (2005) at $25^{\circ} \mathrm{C}$

The 3D visualisation of the images has been obtained through a volume rendering procedure by using the commercial software VGStudio MAX 2.0 (Volume Graphics).

\section{Results and discussion}

\subsection{Rheological properties}

schel-Bulkley model which gave a value of $0.9237 \mathrm{~Pa}$ (with $\mathrm{r}^{2}$ value of 0.8898 ).

\subsection{Secondary liquid-water/saturated sucrose solution}

Two different volumes ( $5 \mu \mathrm{l}$ and $10 \mu \mathrm{l}$ water) of secondary liquid were added to separate suspensions, and scans taken to monitor the progress of the liquid with time. A time lapse of images for the $5 \mu l$ and $10 \mu l$ water droplet added can be seen in Figs. 4 and 5 respectively. For both samples, 9 scans were conducted after different time intervals. For ease of identification, the numbering of scans is in chronological order from 1 to 9 , from the earliest scan conducted to the latest scan. All the scan numbers for the $5 \mu \mathrm{l}$ water droplet end in $\mathrm{A}$, for the $10 \mu \mathrm{l}$ water droplet end in B and for the $10 \mu \mathrm{l}$ saturated sucrose solution end in C. It was not possible to scan both samples at exactly the same time intervals of storage from initial addition of the water, due to the setup of the synchrotron facility. However, attempts were made to keep the times as close as possible to each other for comparison purposes.

The dark region above the suspension is air, as has been highlighted in the first image in Fig. 4. It can be seen when looking at the images from Fig. 4, the $5 \mu l$ water droplet is increasing in size as it progresses through the suspension. This is due to the uptake of sucrose from the suspension by the droplet which results in a volume increase. The time between scans $1 \mathrm{~A}$ and $2 \mathrm{~A}$ is $60 \mathrm{~min}$, and it can be seen during this time period, the droplet has moved approximately $1 \mathrm{~mm}$ into the suspension. Also, the path taken by the water droplet can be seen as a channel of oil is left behind from where the droplet was initially, to where it is now.

The droplet initially takes a spherical shape which is expected for a water droplet. Though, with time the droplet becomes more ellipsoidal (larger in the horizontal diameter, in comparison to the vertical diameter) as can be seen in the image from scan $6 \mathrm{~A}$ after $390 \mathrm{~min}$. The horizontal dimension is larger in comparison to the vertical dimension of the droplet. After $480 \mathrm{~min}$ in scan 7A, the top portion of the droplet slowly moves towards a flat shape and this continues until the upper portion transforms to a concave shape as can be seen in scan $8 \mathrm{~A}$, which was taken after $600 \mathrm{~min}$. The concave shape shows the droplet as it progresses was composed of liquid. This is evident from the top shape, as if it was a solid body it would not deform into this shape. Also, the route taken by the secondary liquid to its position can be seen, when looking at the region above the secondary liquid. After $600 \mathrm{~min}$, the droplet continues spreading axially, as can be seen in scan 9A, taken after $1080 \mathrm{~min}$. Also evident from the scan taken after $1080 \mathrm{~min}$, is some small vertical spreading, to the portion below the droplet.

A similar trend can be seen in Fig. 5 for the time lapse of images for the $10 \mu$ l water droplet, in that the size of the droplet increases as it progresses into the suspension as mentioned for Fig. 4. The droplet is physically bigger than that seen in Fig. 4, which is expected, as the volume added initially is twice the amount. The trend is the same as that seen in Fig. 4 for the $10 \mu \mathrm{l}$ water droplet in Fig. 5, in that initially the droplet takes a spherical shape which then slowly transforms to an ellipsoidal shape with time and finally ends with a flat top with axial spreading.

For the final three scans (scans 7B-9B) taken after $960 \mathrm{~min}$ from the initial addition of the $10 \mu \mathrm{l}$ water droplet, it can be 


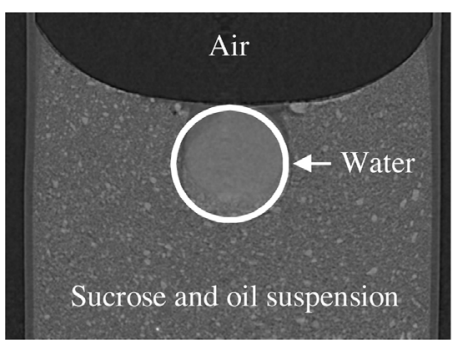

After 15 mins

Scan 1A

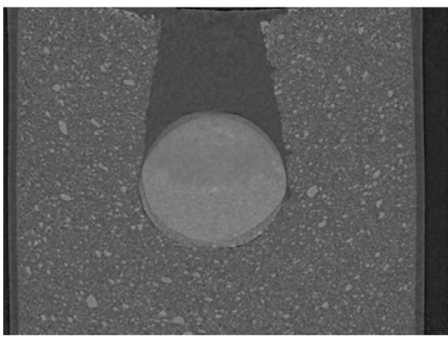

After 240 mins

Scan 4A

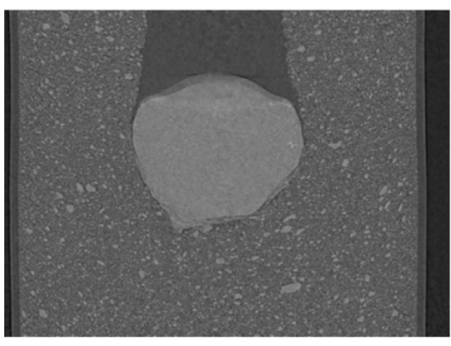

After 480 mins

Scan 7A

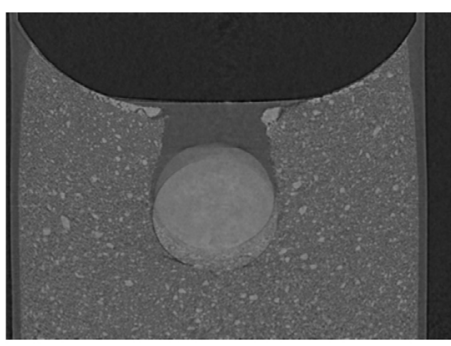

After 75 mins

Scan 2A

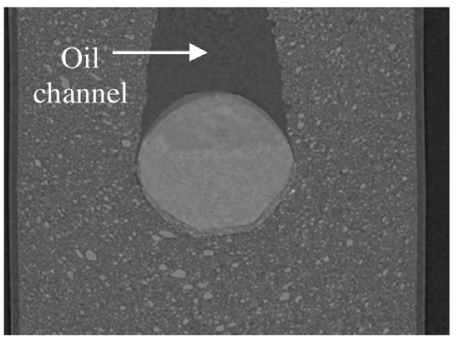

After 330 mins

Scan $5 \mathrm{~A}$

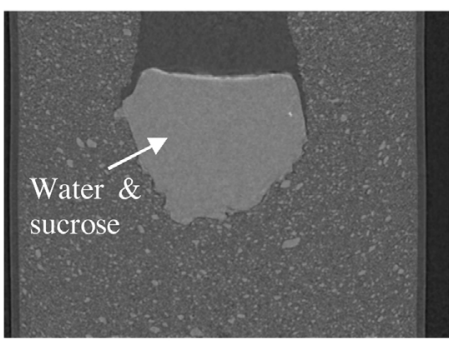

After 600 mins

Scan 8A

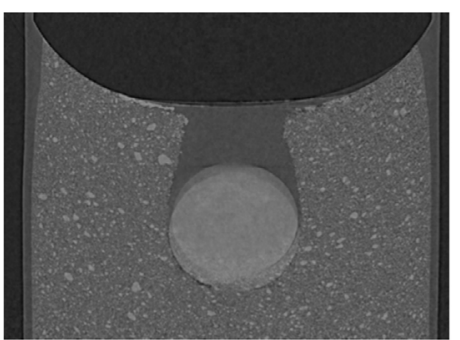

After 120 mins

Scan 3A

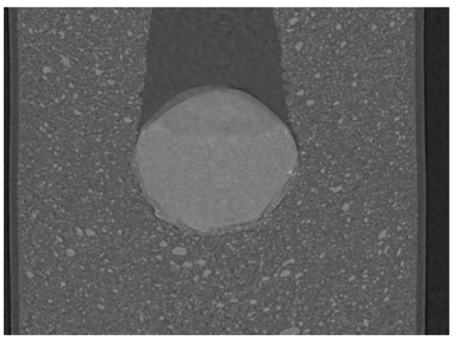

After 390 mins

Scan 6A

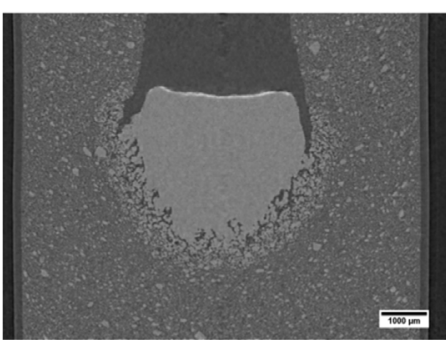

After 1080 mins

Scan 9A

Fig. 4 - Central slice along $X$-axis (where diameter of water droplet is largest), for the $5 \mu l$ water droplet after different storage periods. Diameter of sample holder is $9 \mathrm{~mm}$. Scale on Scan 9A is $1 \mathrm{~mm}$.

seen from these images, there is movement from the right region next to the droplet as the sucrose is pulled towards the droplet, leaving a void of sunflower oil near the wall of the cellulose sample holder. The reason for this was initially believed to be that the droplet was not added perfectly to the centre as can be seen from scan 1B in Fig. 5, when compared to scan $1 \mathrm{~A}$ in Fig. 4. As the droplet moved into the suspension and was larger in volume compared to the $5 \mu \mathrm{l}$ droplet, by scan $6 \mathrm{~B}$ it was very close to one side of the sample holder. Thus by scan 7B, the attraction towards the droplet for the sucrose particles from the bulk suspension is strong, resulting in the sucrose particles moving towards the droplet (as a complete network). This was not the case for the sucrose particles within the suspension to the left in scans 7B-9B. It can be seen for this section; no major movement towards the droplet is seen. For this reason, attempts were made to avoid the water droplet coming into contact with the cellulose sample holder, to ensure no external influence upon the movement of the droplet.

For the first 3 images shown in Fig. 4, for scans taken up to $120 \mathrm{~min}$ after the initial addition of the water droplet, there is a shadow effect around the droplet. The reason for this is the movement of the droplet during the duration of the scan.
Therefore, the water droplet location for the earlier projections is different when compared to the later projections. Consequently, when the projections are reconstructed, this leads to the shadow effect being present, in the final reconstructed images. Ideally, when scanning samples using X-ray CT, they should remain completely still without any movement as projections are taken as the sample is rotated. However, for the system being studied in this case, it is not possible to achieve this. For this reason, efforts were taken with preliminary scans to produce a sufficiently quick scan to capture the dynamics of the system with satisfactory resolution. For the later scans between $240-480 \mathrm{~min}$ after the addition of $5 \mu \mathrm{l}$ water droplet, the shadow effect is less, indicating that the droplet movement is now slower. This is expected as the droplet continues to take up sucrose from the bulk suspension at a slower rate. As the water droplet movement is slower, it is expected the change in location between projections of the droplet during a scan will be reduced, and therefore the shadow effect in the final reconstructed image will be less evident. For the final 2 scans taken after 600 and $1080 \mathrm{~min}$ in Fig. 4, there is no shadow as now the speed of the droplet movement has significantly decreased. Though, it can be seen that between scan $8 \mathrm{~A}$ and 


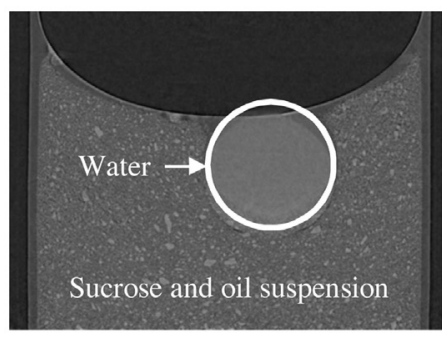

After 0 min Scan 1B

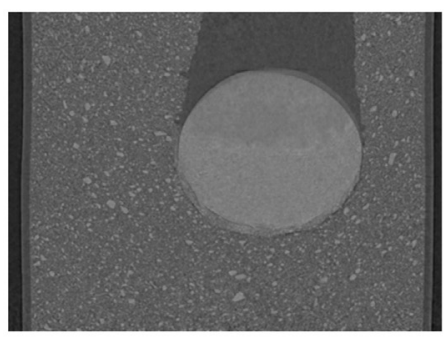

After 360 mins

Scan 4B

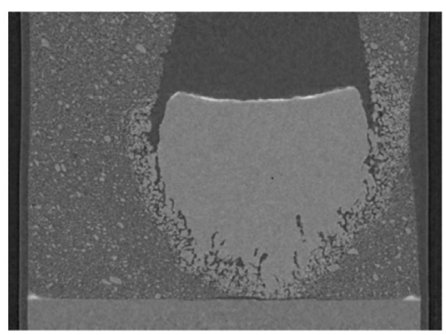

After 960 mins

Scan 7B

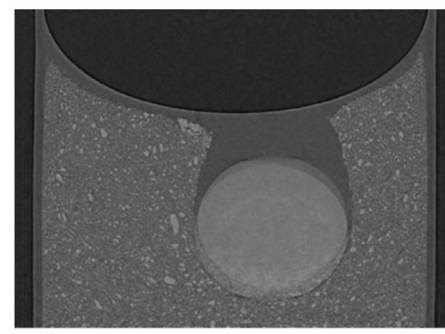

After 105 mins

Scan 2B

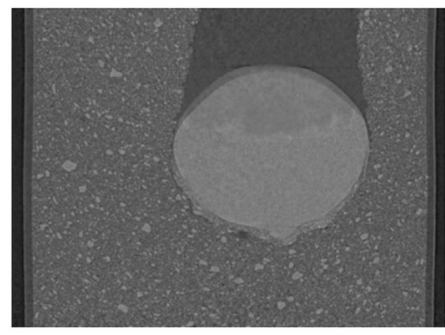

After 420 mins

Scan 5B

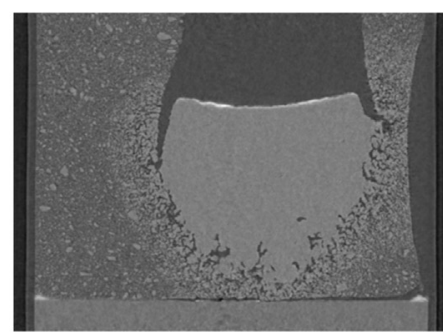

After 1020 mins

Scan 8B

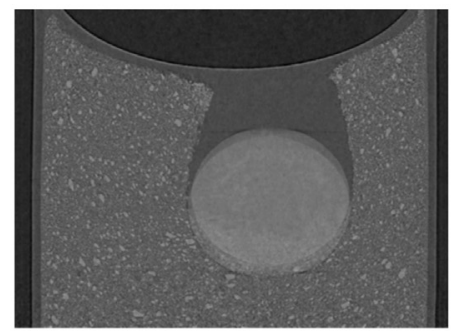

After 140 mins Scan 3B

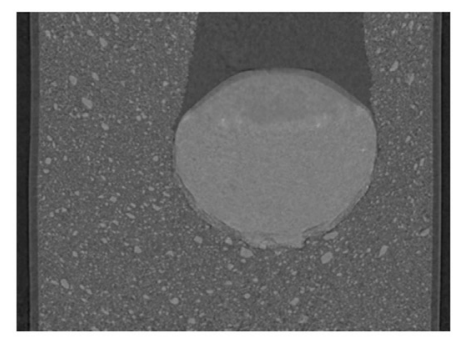

After 500 mins Scan 6B

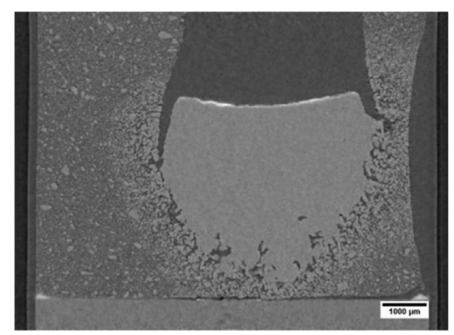

After 1740 mins Scan 9B

Fig. 5 - Central slice along $X$-axis (where diameter of water droplet is largest), for the $10 \mu l$ water droplet after different storage periods. Diameter of sample holder is $9 \mathrm{~mm}$. Scale on Scan 9 B is $1 \mathrm{~mm}$.

9A, the secondary liquid movement is still occurring, though now very different to that seen initially from the earlier scans.

During the scan as seen in Figs. 4 and 5, the droplet is moving with time further into the suspension. Therefore, to follow the droplet, the portion of the sample being scanned was changed between scans when needed to ensure the droplet was within the field of view. For example in Fig. 4, between scan $1 \mathrm{~A}$ and scan $6 \mathrm{~A}$, the portion scanned was changed along the height (y-axis in Fig. 2) of the sample, to ensure the region of interest which in this case was the water droplet was in complete view for the portion being scanned. This can schematically be seen in Fig. 6. Similar changes were made to the scans for the $10 \mu \mathrm{l}$ water droplet in Fig. 5, to ensure the droplet movement was tracked.

The images shown in Fig. 4 show the movement of the droplet along the X-axis in relation to the axis shown in Fig. 2. Using the software VGStudio MAX2.0, 3D volume renderings were produced to show the sample along the different planes. Fig. 7 is a volume rendering for scan 9A from the images in Fig. 4. The oil phase has been masked out, leaving the sucrose and water/sucrose phase. A crater-like surface on the top of the water/sucrose mixture is evident, which is believed to be due to the sucrose particles. The water alone is not able to take and deform into such a shape within the liquid state, indicating now the droplet is in a solid or semi-solid state.

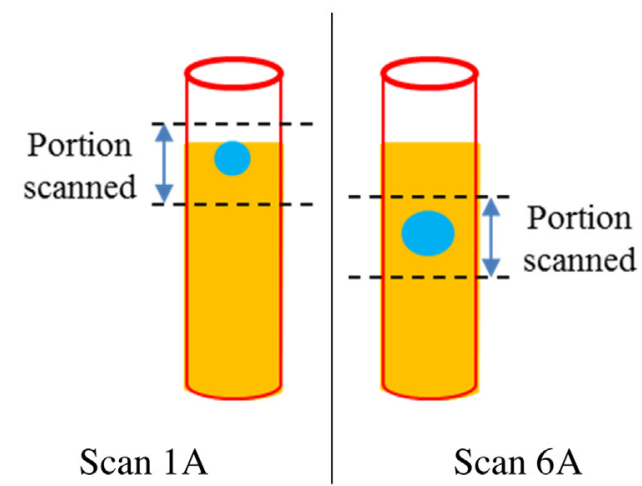

Fig. 6 - Schematic for the change in field of view scanned for samples, to follow the progression of secondary liquid.

Also, near the top of the water/sucrose mixture, it can be seen there is a separation between the droplet and the bulk suspension, which was occupied by oil, as well as the spreading of the water along the different planes. This indicates both the movement of sucrose into the droplet as well as spreading of water from the droplet into the bulk suspension.

A similar volume rendering was done of scan 9B from Fig. 5 which can be seen in Fig. 8. Image (a) shows how far the water has spread along the different planes, similar to that shown in 


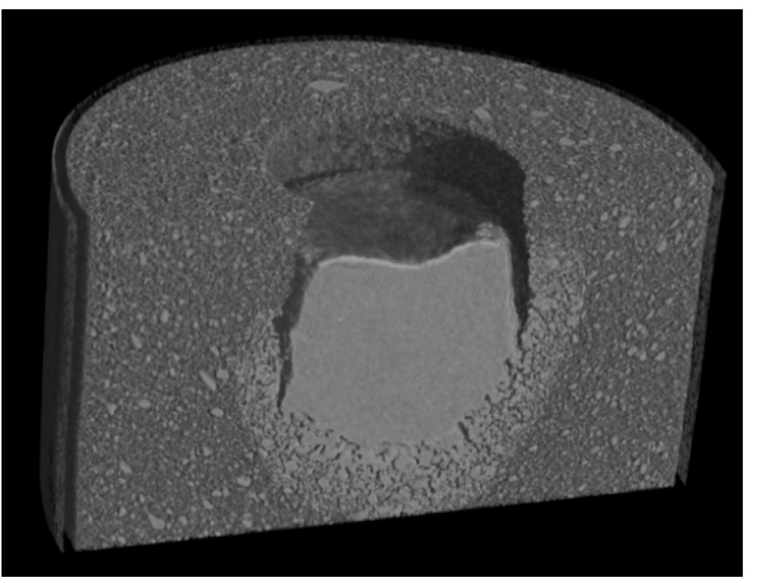

Fig. 7 - Volume rendering of Scan 9A from Fig. 4, cut along the $X$ and $Y$ plane with the removal of the oil phase to highlight the top surface of the sucrose/water droplet.

Fig. 7 for the $5 \mu$ l water droplet. In image (b) again the oil phase has been masked out, with a volume of interest focusing on the sucrose/water droplet. A similar crater surface on the top of the water/sucrose shown in Fig. 7 can be seen, which is believed to be due to the sucrose particles. The sucrose/water droplet body appears consistent in greyscale value, indicating that the body is uniform in its composition throughout.

The images from Fig. 4 were adjusted with false colours using a look up table in image $J$ (look up table option-BRGBCMYW) to better enhance the contrast within the water droplet which can be seen in Fig. 9. For scan 1A in Fig. 9 it can be seen that the whole droplet is dark blue to indicate it is predominantly water with little solid sucrose which is expected after $15 \mathrm{~min}$ from addition. By scan 3B, the lower portion of the droplet is turning slightly towards a lighter blue colour, as solid sucrose begins to enter from the lower region of the droplet, as the droplet progresses into the suspension. This lower portion of light blue continues to grow from scan 4A to $6 \mathrm{~A}$, progressively coming closer to the top as time progresses. By scan 7A the whole droplet is of a fairly uniform colour of both light and dark blue which is similar to scan 8A \& 9A, indicating now that the initial water droplet contains sucrose all throughout the droplet. A similar trend can be seen in Fig. 10 for the adjusted images for the $10 \mu \mathrm{l}$ water droplet from Fig. 5 . For scan 9B in Fig. 10, it can be seen in the area surrounding the droplet, the concentration of dark blue is more. This is due to the sucrose particles, which are attracted to the water and come together to reach the droplet.

From the images presented in Figs. 9 and 10, it is believed the sucrose enters into the water droplet from the lower portion of the droplet. This results in the volume increase for the droplet seen within the time lapse of images presented. A schematic of the method for how this occurs can be seen in Fig. 11.

In stage 1 after initial addition, the droplet contains no sucrose and is composed solely of water, which corresponds to scan $1 \mathrm{~A}$ and $1 \mathrm{~B}$ from Figs. 9 and 10. By stage 2, the water droplet has moved a little into the bulk suspension, with solid sucrose from the bulk suspension entering into the lower portion of the droplet. This causes a slight increase in volume of the droplet, which corresponds to scan 3A from Fig. 9. By stage 3 , the droplet has moved further into the suspension with additional sucrose entering into the droplet from the suspension. As well as this, some of the solid sucrose which had previously entered in stage 2 has now dissolved, which corresponds to scan 4A from Fig. 9. Also, in stage 3, the droplet begins to transform from a spherical shape seen in stage 2 to a more ellipsoidal shape as the horizontal diameter increases quicker in comparison to the vertical diameter, due to the area of contact of sucrose from the bulk suspension with the droplet.

Then after a long time from the initial addition in stage 4 in Fig. 11, the droplet has progressed even further into the bulk suspension, with dramatic changes in the shape of the droplet. The droplet is no longer ellipsoidal as that seen in stage 3 and now has transformed into a distinct irregular shape, due to how solid sucrose entered into the droplet as it progressed. Also, now the droplet is fully occupied by sucrose which corresponds to scan $8 \mathrm{~A}$ from Fig. 9.

After stage 4 in Fig. 11, now the droplet cannot accept any further solid sucrose as it is fully occupied by sucrose as it can feasibly take. Therefore, now the movement direction is of the water or water sucrose solution mixture away and into the bulk suspension, to come into contact with further solid sucrose within the bulk suspension. This is highlighted as stage 5 in Fig. 11, as further spreading can be seen in scan 9A from Fig. 9, which demonstrates the system has not yet reached equilibrium and further mass transport mechanisms are taking place to drive the movement of the secondary liquid. Thus, the shape of the droplet is changing further with spreading of the secondary liquid into the bulk suspension.

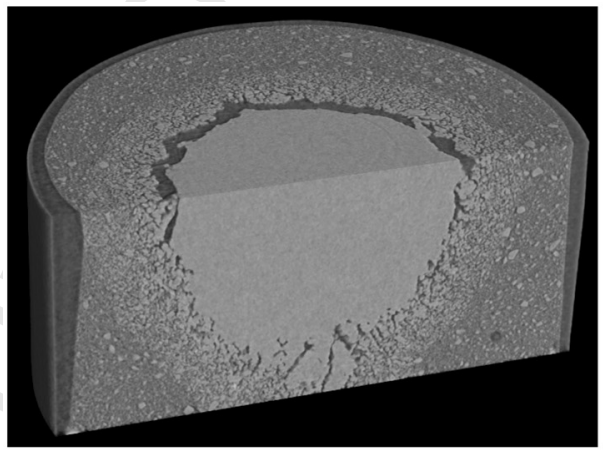

(a)

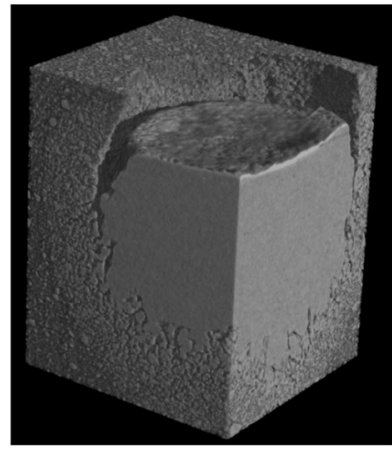

(b)

Fig. 8 - Volume rendering of Scan 9 B from Fig. 5; (a) cut along the $X$ and Y plane focusing on the droplet; (b) cut along the $X$ and $Y$ plane with the removal of the oil phase to highlight the top surface of the sucrose/water droplet. 


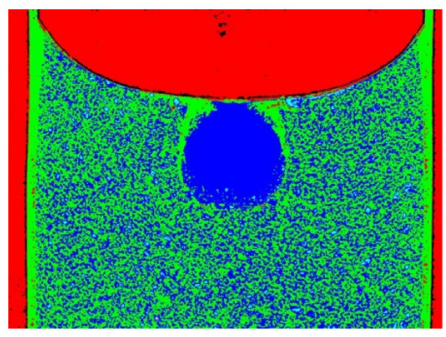

After 15 mins Scan 1A

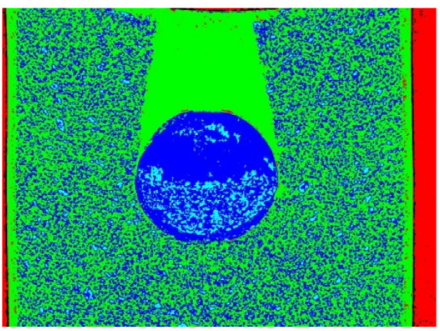

After 240 mins

Scan 4A

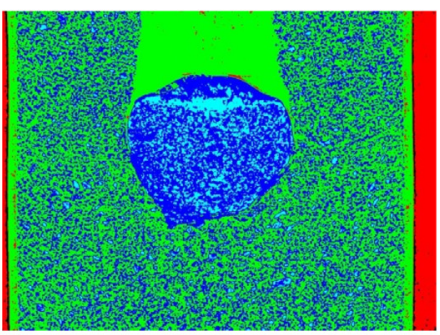

After 480 mins

Scan 7A

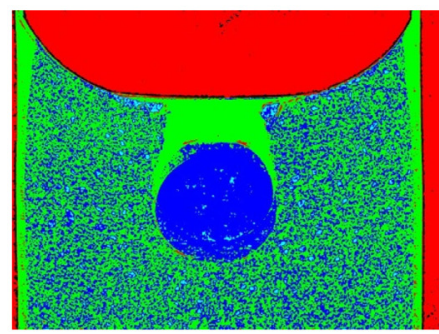

After 75 mins Scan 2A

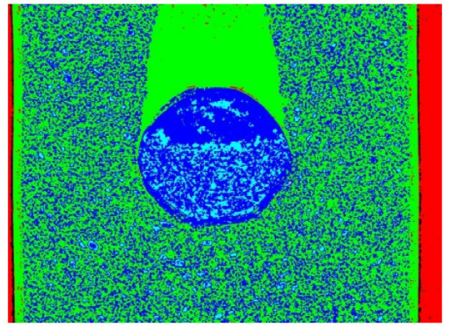

After 330 mins

Scan 5A

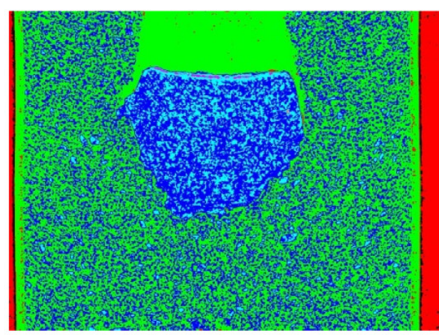

After 600 mins

Scan 8A

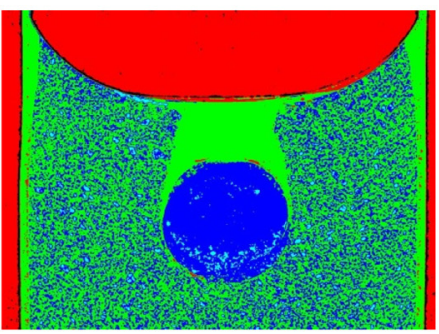

After 120 mins

Scan 3A

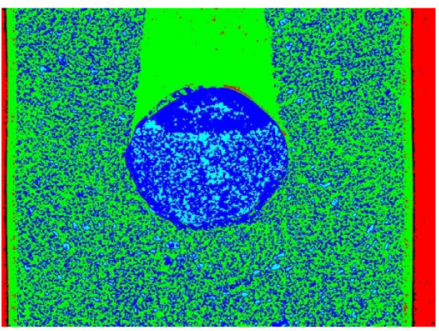

After 390 mins

Scan 6A

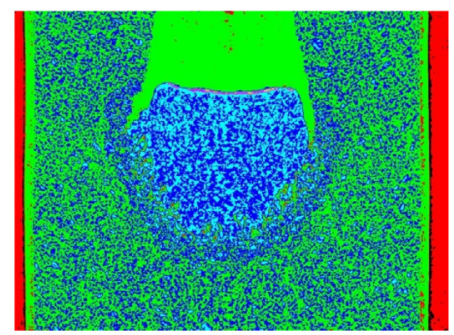

After 1080 mins

Scan 9A

Fig. 9 - Central slice along $X$-axis (where diameter of water droplet is largest), for $5 \mu l$ water droplet after different storage periods. Same images as in Fig. 4.

Similar comparisons can be made for the images for the $10 \mu \mathrm{l}$ water droplet from Fig. 10, to correlate to the different stages shown schematically in Fig. 11. This shows how initially the movement is of the sucrose from the bulk suspension into the water droplet, as long as the water droplet can take up the sucrose. However, after the droplet is fully occupied by sucrose, the movement is of the water away from the bulk water into the suspension, to allow it to come into contact with further sucrose from the bulk suspension.

For the sample to which $10 \mu$ l of saturated sucrose solution was added, a time lapse of images for this sample can be seen in Fig. 12. It can be seen for this sample in scan 1C, the droplet is very stable with no shadow around it. The reason for this is the droplet is not moving significantly in comparison to the scan duration of $24 \mathrm{~min}$. After $600 \mathrm{~min}$ in scan 2C, it can be seen that the droplet has only moved $0.3 \mathrm{~mm}$ from the surface of the suspension with some settling of the sucrose particles within the suspension. By scan 3C after $1250 \mathrm{~min}$, the saturated sucrose droplet has only moved $0.4 \mathrm{~mm}$ from the surface of the suspension, with further settling of the sucrose particles within the bulk suspension. The reason for very minimal movement of the droplet is believed to be due to the inability of the droplet to take up and dissolve any further sucrose, as it is already saturated with sucrose. This therefore limits the mobility of the saturated sucrose solution droplet within the suspension. This is very different behaviour when compared to that of the $10 \mu$ l of water droplet in Fig. 5.

\subsection{SEM of sucrose/water mixture}

The sucrose/water mixture from Fig. 5 after 1740 min (schematically represented as stage 5 in Fig. 11) was removed and it was found to be solid in composition. This was cleaned with hexane to remove any oil residue, leaving behind only the sucrose/water skeleton network (Killian and Coupland, 2012). This was then investigated under the SEM and corresponding images can be seen in Fig. 13. A magnified image is reported on the right of Fig. 13, where the presence of sucrose crystals bound with solid bridges can be seen. It is believed these bridges formed as water came into contact and partially dissolved sucrose and then recrystallized. This agrees with the work carried out by Killian and Coupland (2012) who used model suspensions of soybean oil and sucrose with water addition. The samples with water were able to resist structural changes due to elevated temperature (maintaining shape and structure), due to the matrix network formation of sucrose and water in comparison to samples without water incorporated. 


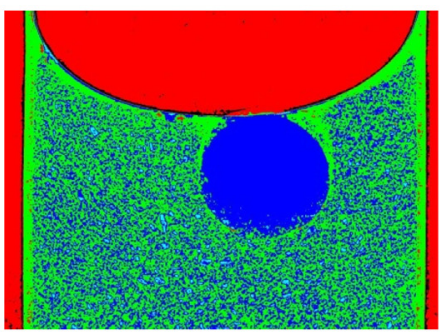

After $0 \mathrm{~min}$

Scan 1B

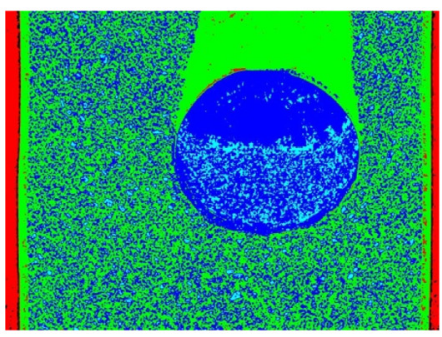

After 360 mins

Scan 4B

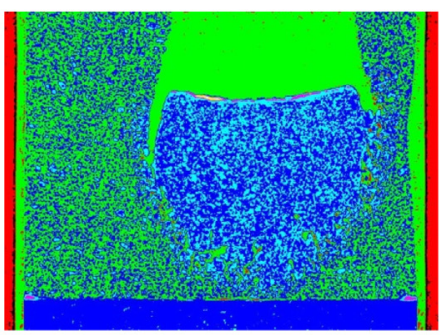

After 960 mins Scan 7B

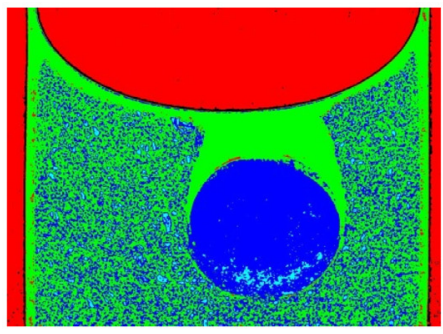

After 105 mins

Scan 2B

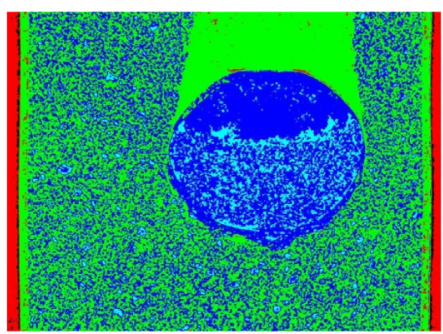

After 420 mins

Scan 5B

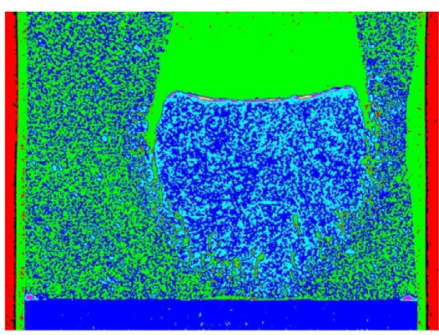

After 1020 mins

Scan 8B

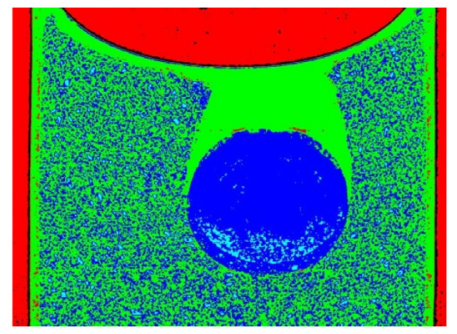

After 140 mins

Scan 3B

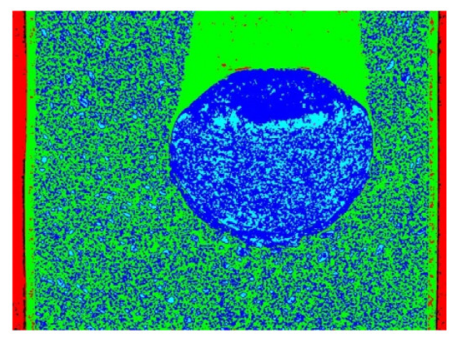

After 500 mins

Scan 6B

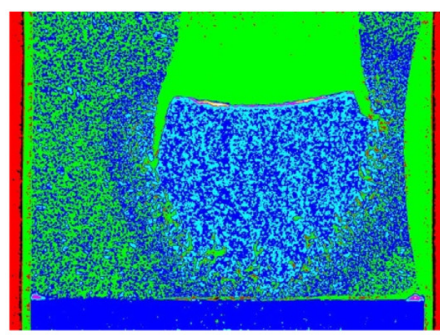

After 1740 mins

Scan 9B

Fig. 10 - Central slice along $\mathrm{X}$-axis (where diameter of water droplet is largest), for $10 \mu \mathrm{l}$ water droplet after different storage periods. Same images as in Fig. 5.

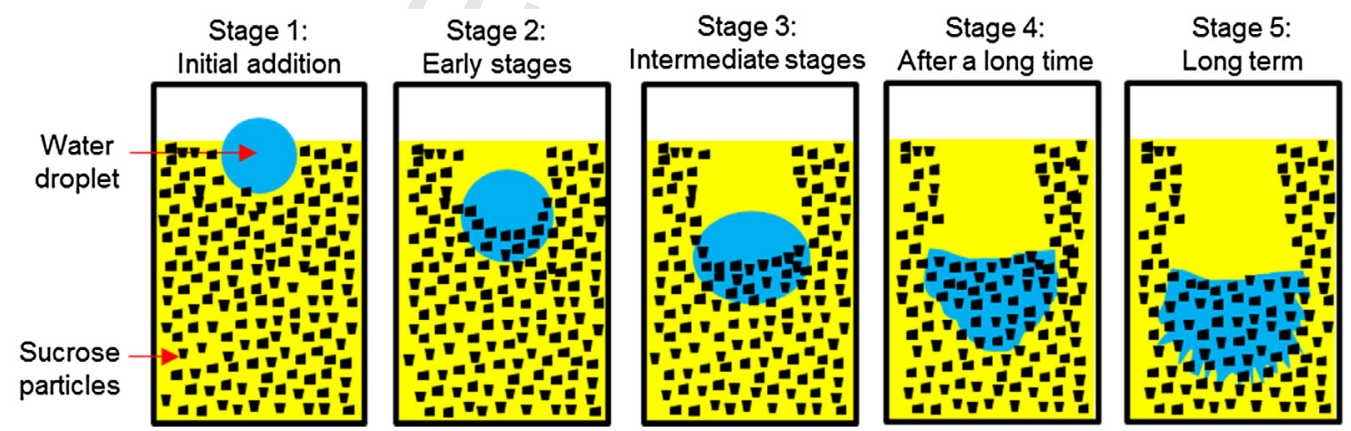

Fig. 11 - Schematic for the stages of movement for the secondary liquid.

This was ascribed to the liquid bridge formation due to the presence of water.

\subsection{Droplet displacement}

The displacement of the droplet with time (from the surface of the suspension to the top of the droplet) corresponding to images shown in Figs. 4, 5 and 12 is represented in Fig. 14 for the $5 \mu \mathrm{l}$ water droplet, $10 \mu \mathrm{l}$ water droplet and $10 \mu \mathrm{l}$ saturated sucrose droplet.
The saturated sucrose droplet (denser than water), moves a distance of approximately $0.3 \mathrm{~mm}$ over $1200 \mathrm{~min}$. This is due to settling of the particles rather than gravitational effects as can be seen from the images in Fig. 12, with the small layer of oil forming on the surface. If the penetration was driven by gravitational effects, the saturated sucrose droplet would have travelled quicker which, as it can be observed from Fig. 14, is not the case.

For the $5 \mu \mathrm{l}$ and $10 \mu \mathrm{l}$ water droplets, over the first $500 \mathrm{~min}$, the displacement with time is fairly consistent. This is 


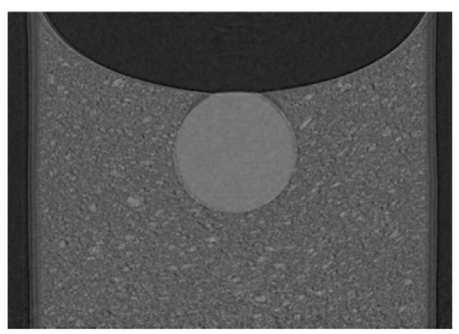

After 15 mins Scan 1C

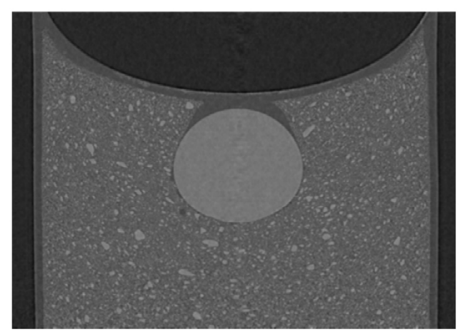

After 600 mins Scan $2 \mathrm{C}$

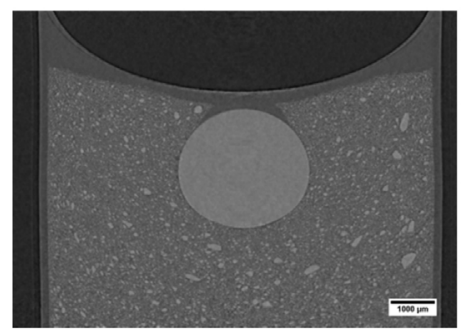

After 1250 mins Scan 3C

Fig. 12 - Central slice along $X$-axis (where diameter of water droplet is largest), for the $10 \mu l$ saturated sucrose droplet after different storage periods. Diameter of sample holder is $9 \mathrm{~mm}$. Scale on Scan 3C is $1 \mathrm{~mm}$.

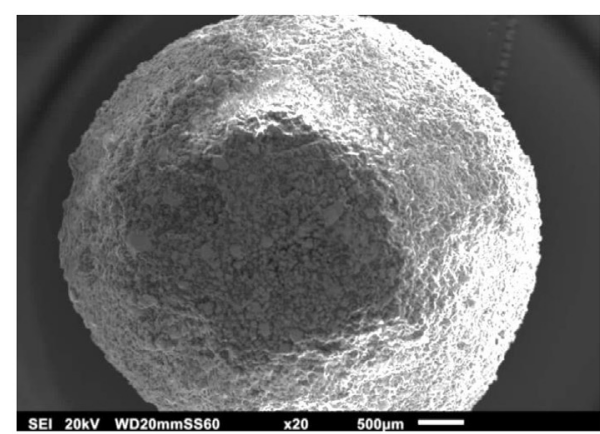

Low magnification

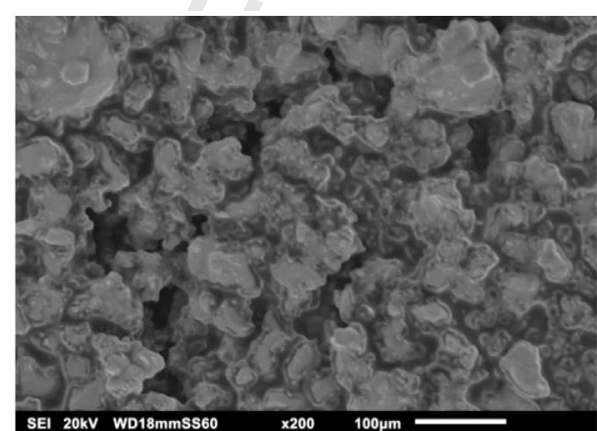

High magnification

Fig. 13 - SEM image of sucrose/water mixture.

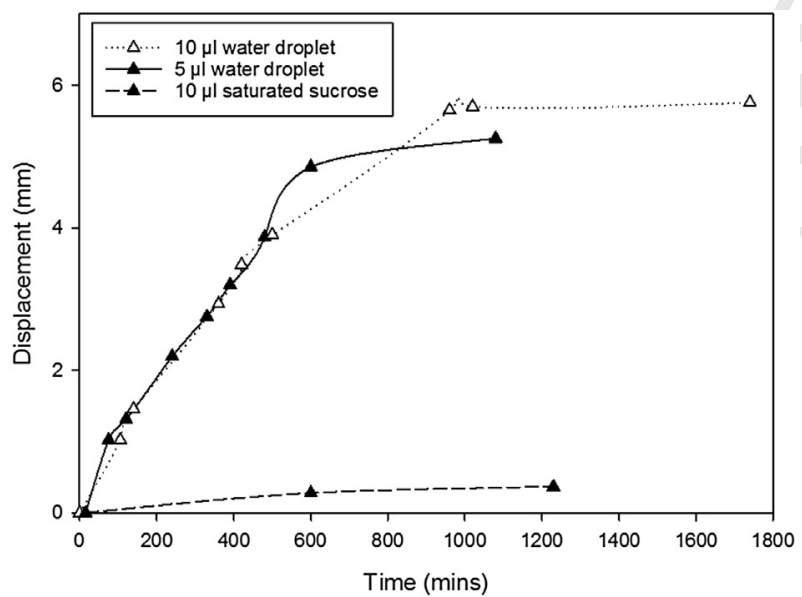

Fig. 14 - Displacement with time for droplets from images in Figs. 4, 5 and 12. believed to be due to the ability of the water droplets to take up sucrose from the bulk suspension. However, after $500 \mathrm{~min}$, the smaller volume droplet $(5 \mu \mathrm{l})$ is nearly full, whereas the larger volume droplet $(10 \mu \mathrm{l})$ still has the capacity to take up further sucrose and therefore continues to advance into the suspension. After $1000 \mathrm{~min}$, the larger volume water droplet is no longer able to progress into the suspension as it is fully occupied with as much sucrose as it can take up from the bulk suspension.

\subsection{Sucrose concentration}

The relative volume increase for the different water droplets used, 5 \& $10 \mu$ l, whose images are shown respectively in Figs. 4 and 5 is reported in Fig. 15. If the increase in volume

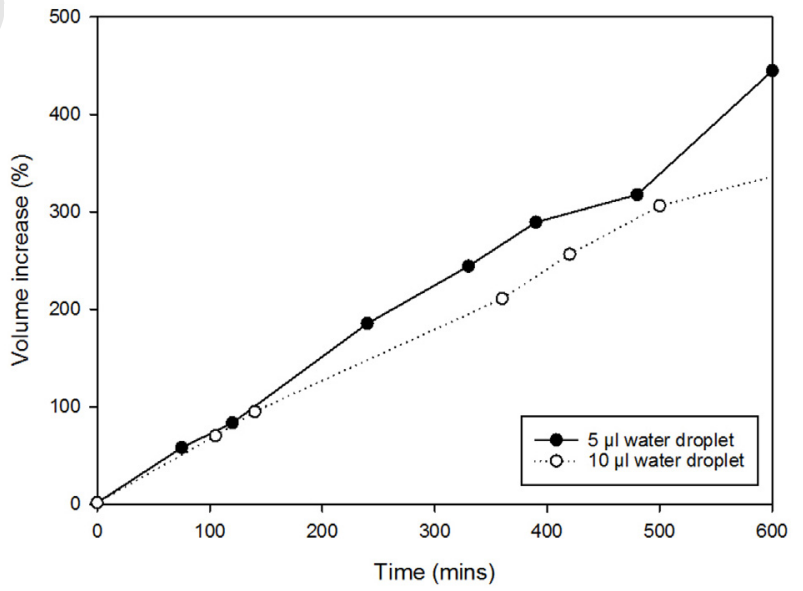

Fig. 15 - Volume increase of droplet with time for images from Figs. 4 and 5.

shown in Fig. 15 is due to the mass transfer of solid sucrose from the surrounding suspension into the secondary liquid droplet, and assuming there is no mass transfer or diffusion of the oil phase into the droplet, it is possible to calculate the concentration of sucrose within the droplet with time. At $25^{\circ} \mathrm{C}$, the solubility of sucrose is $2.0741 \mathrm{~g}$ of sucrose per $\mathrm{g}$ of water, which corresponds to a $67.47 \%$ wt concentration of sucrose (Bucke, 1995). Also, from the literature it is known, that the volume is conserved as solid sucrose is dissolved in water (HirschmÜLler, 2013). The difference between the theoretical volume increase and the actual volume increase is less than $0.35 \%$, and approaches $0 \%$ when moving towards a saturated sucrose solution (HirschmÜLler, 2013). Using the information that the overall volume is conserved, it is possible to track the increase in the volume of the water droplet as sucrose enters, 


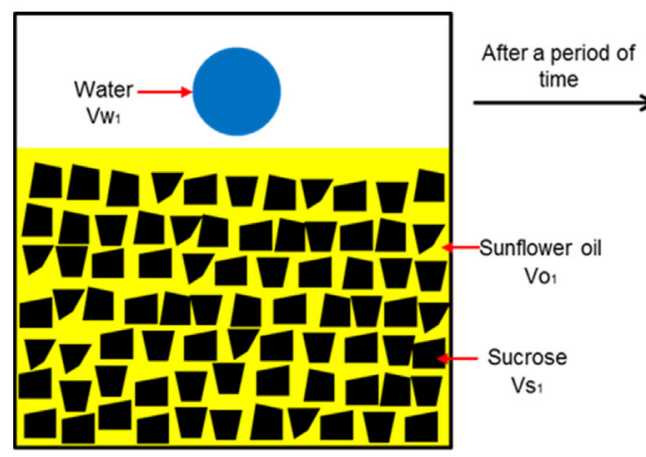

$T_{1}=$ time 0

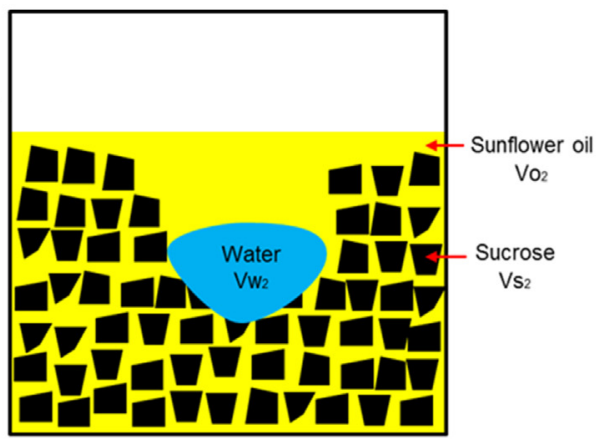

$T_{2}=$ after some time

Fig. 16 - Schematic of tracking the change in volume of the water droplet within the system with time.

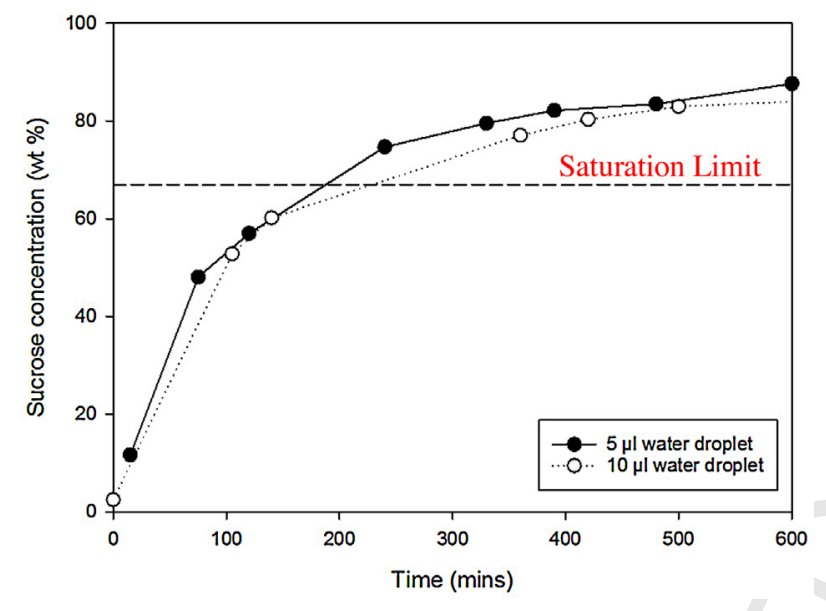

Fig. 17 - Sucrose concentration within the droplet with time for images in Figs. 4 and 5.

and correlate this back to the sucrose concentration (knowing the density of sucrose, $1588 \mathrm{~kg} / \mathrm{m}^{3}$ (Bucke, 1995)) within the droplet with time.

The change in volume due to the sucrose entering the water droplet is shown schematically in Fig. 16. At time 0, before the addition of the water droplet, the volume of the water $\mathrm{Vw}_{1}$, volume of sunflower oil $\mathrm{Vs}_{1}$ and the volume of sucrose $\mathrm{Vs}_{1}$ within the system known. Then after a period of time, as the water droplet progresses into the suspension, the water droplet volume increases to $\mathrm{Vw}_{2}$, due to the movement of sucrose from the bulk suspension into the water droplet. The total amount of sucrose within the system is still the same; however, the amount of sucrose within the surrounding bulk suspension $\mathrm{Vs}_{2}$ is now less than $\mathrm{Vs}_{1}$ as some sucrose has moved into the water droplet. The sunflower oil volume $\mathrm{Vo}_{2}$ is the same as $\mathrm{Vo}_{1}$ and the location of the oil does not change.

Focusing only on the water droplet from Fig. 16, the increase in volume of the water droplet with time as sucrose enters the water droplet can be used to calculate the sucrose concentration of the droplet. The change in sucrose concentration within the droplet can be seen in Fig. 17.

It can be observed that the sucrose concentration of the droplet goes above the saturation limit of $67 \%$ wt at $25^{\circ} \mathrm{C}$ (black dashed line in Fig. 17 with no data points), indicating that the droplet must contain both dissolved and solid sucrose. After approximately $200 \mathrm{~min}$, there is sufficient sucrose within the droplet to saturate it; however, the movement of further solid sucrose from the bulk suspension into the droplet con-

$\begin{aligned} & \text { Table } \mathbf{2} \text { - Surface area to volume ratio for droplets based } \\
& \text { on volume of a sphere. }\end{aligned}$
\begin{tabular}{ll} 
Volume of droplet $(\mu \mathrm{l})$ & Surface area to volume ratio $\left(\mathrm{mm}^{-1}\right)$ \\
\hline 5 & 2.82 \\
10 & 2.24 \\
\hline
\end{tabular}

tinues. Also, the smaller volume droplet $(5 \mu \mathrm{l})$ is increasing in sucrose concentration at a quicker rate in comparison to the larger volume droplet $(10 \mu \mathrm{l})$, indicating the uptake rate of sucrose is quicker for the smaller volume droplet. This is believed to be due to the surface area to volume ratio of the droplets (Table 2 for a sphere, the surface area to volume ratio is $3 /$ radius). Therefore, for the smaller volume droplet $(5 \mu \mathrm{l})$ can take up solid sucrose at a faster rate in comparison to the larger volume droplet $(10 \mu \mathrm{l})$.

Moreover, as the secondary liquid droplet progresses into the suspension, the viscosity and density change due to solid sucrose entering into the droplet. The effect of increasing the concentration of sucrose solution upon the viscosity was measured (\% relates to mass fraction) which can be seen in Table 1.

For the system being studied, it would appear gravitational sedimentation is not driving the movement of the secondary liquid. In fact, the denser saturated sucrose solution moves considerably slower than the water droplet, even though the saturated sucrose solution is denser. Therefore, this seems to indicate other transport mechanisms are driving the movement.

\subsection{Mean grey value}

It can be seen that there is a clear change in the greyscale of the droplet in the time lapse of images shown in Figs. 4 and 5, respectively for the $5 \mu \mathrm{l}$ and $10 \mu \mathrm{l}$ water droplets, as they progresses through the suspension, whereas, for the $10 \mu$ l saturated sucrose droplet (see Fig. 12), there is only a minimal change. The ImageJ software was used to track the change in grey value with time for the images shown. These are 16 bit images, with a grey range of 0-65,535, where 0 corresponds to completely black and 65,535 to completely white and in between are shades of grey.

The area of the secondary liquid droplet chosen to measure the greyscale within images Figs. 4, 5 and 12 was in the whole droplet, while avoiding the shadow areas present at the outer regions of the droplet, due to the movement during the scan. A plot of the mean grey values for all images with time can 


\section{Table 3 - Mean grey value for droplets from images in Figs. 4, 5 and 12 (error is standard deviation).}

\begin{tabular}{lccl} 
Scan number & $5 \mu l$ water (Fig. 4) & $10 \mu l$ water (Fig. 5) & 10 $\mu$ l saturated sucrose (Fig. 12) \\
\hline 1 & $28420 \pm 1110$ & $28280 \pm 960$ & $30100 \pm 830$ \\
2 & $30110 \pm 1320$ & $30120 \pm 1390$ & $30210 \pm 880$ \\
3 & $30610 \pm 1180$ & $30580 \pm 1290$ & $30360 \pm 890$ \\
4 & $31530 \pm 1360$ & $31590 \pm 1330$ & - \\
5 & $31750 \pm 1510$ & $31810 \pm 1350$ & - \\
6 & $31930 \pm 1420$ & $32010 \pm 1280$ & - \\
7 & $32204 \pm 1340$ & $32340 \pm 1290$ & - \\
8 & $32240 \pm 1204$ & $32380 \pm 1340$ & - \\
9 & $32510 \pm 1170$ & $32490 \pm 1170$ & \\
\hline
\end{tabular}

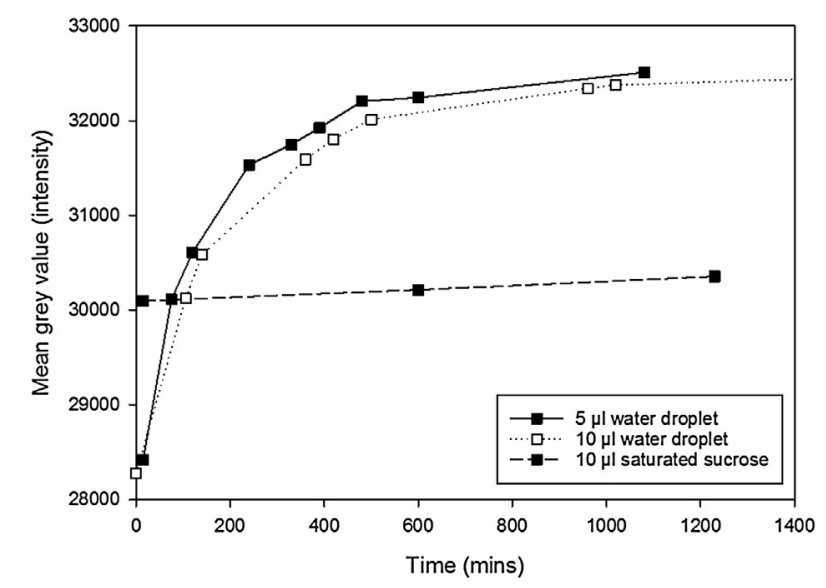

Fig. 18 - Mean grey value for droplets from images in Figs. 4, 5 and 12. be seen in Fig. 18 while the actual values are listed in Table 3 For the saturated sucrose droplet, the droplet has an initial mean grey value of 30,100 after addition, increasing to 30,350 after $1200 \mathrm{~min}$. Also, the standard deviations for the saturated sucrose droplet give an indication that the droplet is of relatively uniform grey value, which is expected as the droplet is already saturated.

For the water droplets (both $5 \mu \mathrm{l} \& 10 \mu \mathrm{l}$ ), it can be seen there is a clear increase with time of the mean grey value (indicating the droplet is becoming brighter) with time as the droplets progress into the bulk suspension and takes up solid sucrose. This is in agreement with the images from Figs. 4 and 5, where the sucrose particles within the suspension have a higher grey value in comparison to the oil and water. Thus, when the droplet takes up sucrose from the suspension, this will lead to a change in the grey value of the droplet towards a higher value.

The mean grey value for scan 1A in Fig. 4 is approximately 28,400 , as can be seen in Fig. 18. After $60 \mathrm{~min}$ h from scan $1 \mathrm{~A}$ for scan $2 \mathrm{~A}$, the mean grey value had increased to 30,100 which results in a significant increase in the mean grey value. The mean grey value now is similar to the saturated sucrose solution, though from Fig. 17, it is known that there is not sufficient solid sucrose within the droplet to saturate it. This indicates that the sucrose present within the droplet is likely to be in a solid state, as this would account for the higher grey value to give an average similar to the saturated sucrose solution. After $120 \mathrm{~min}$ (scan 3A), there is a small increase in the mean grey value to 30,600 . After $240 \mathrm{~min}$ (scan $4 \mathrm{~A}$ ), the mean increases considerably to 31,500 . After $330 \mathrm{~min}$ (scan $5 \mathrm{~A}$ ), the mean increases slightly to 31,800 . Scans $7 \mathrm{~A}$ and $8 \mathrm{~A}$ have a similar mean grey value of 32,200 , as the scans were taken close to each other, after $480 \mathrm{~min}$ and $600 \mathrm{~min}$ respectively. Finally, scan 9A has the highest mean grey value of 32,500 after $1080 \mathrm{~min}$. This is expected, as this scan was the last to be taken, and will contain the most sucrose within the droplet and therefore have the highest mean grey value. A similar change in grey value is seen for the $10 \mu \mathrm{l}$ water droplet for the images in Fig. 5 which can be seen in Fig. 18. The final mean grey values measured for both the $5 \mu l$ and $10 \mu l$ water droplets are much higher in comparison to the mean grey value of the saturated sucrose droplet from Fig. 12, indicating the composition is not of a saturated sucrose solution.

The smaller volume water droplet $(5 \mu \mathrm{l})$ always has a higher mean grey value than the larger volume water droplet $(10 \mu \mathrm{l})$, which indicates a greater amount of sucrose being present within the droplet. This is believed to be due to the surface area to volume ratio as mentioned previously, allowing the smaller droplet to take up sucrose at a quicker rate in comparison to the larger volume droplet. Also, from Table 3 it can be seen that the standard deviations with time for the water droplets are greater in comparison to the saturated sucrose droplet, indicating a more varied grey value for the droplet, which could be attributed to the presence and absence of solid sucrose in different regions of the droplet.

During the scans of the sample, the flux and X-ray beam energy can vary slightly with time. To ensure this did not influence the absorbance and therefore the grey values of the different materials within the sample, the grey value of the air for all samples was measured, which if consistent should have a consistent grey value. The images shown in Figs. 4, 5 and 12 were cropped to remove the air from the side of the sample which is of no interest for analysis purposes. This area to the left of the sample was measured to see the consistency of the grey value of the air. For the images from Fig. 4 for the $5 \mu l$ water droplet, the mean grey value of the air for scans $1 \mathrm{~A}-9 \mathrm{~A}$ was in the range of 9997-10,082, with an average of 10,046, indicating a fairly consistent grey value for the air with minimal fluctuation. For the $10 \mu \mathrm{l}$ water droplet from Fig. 5, the air grey value was in the range of 9995-10,098 with an average of 10,036 which is similar to the $5 \mu \mathrm{l}$ water droplet. Similar grey values were recorded for the air in the sample for the $10 \mu \mathrm{l}$ saturated sucrose droplet from Fig. 12.

\subsection{Greyscale changes within droplet}

Fig. 19(i) is an enlarged image of scan 5A in Fig. 4. It can be seen that the top portion of the droplet has a different grey value in comparison to the bottom portion. The reason for this is believed to be, as mentioned previously, that as the droplet is progressing through the suspension, the lower portion of 


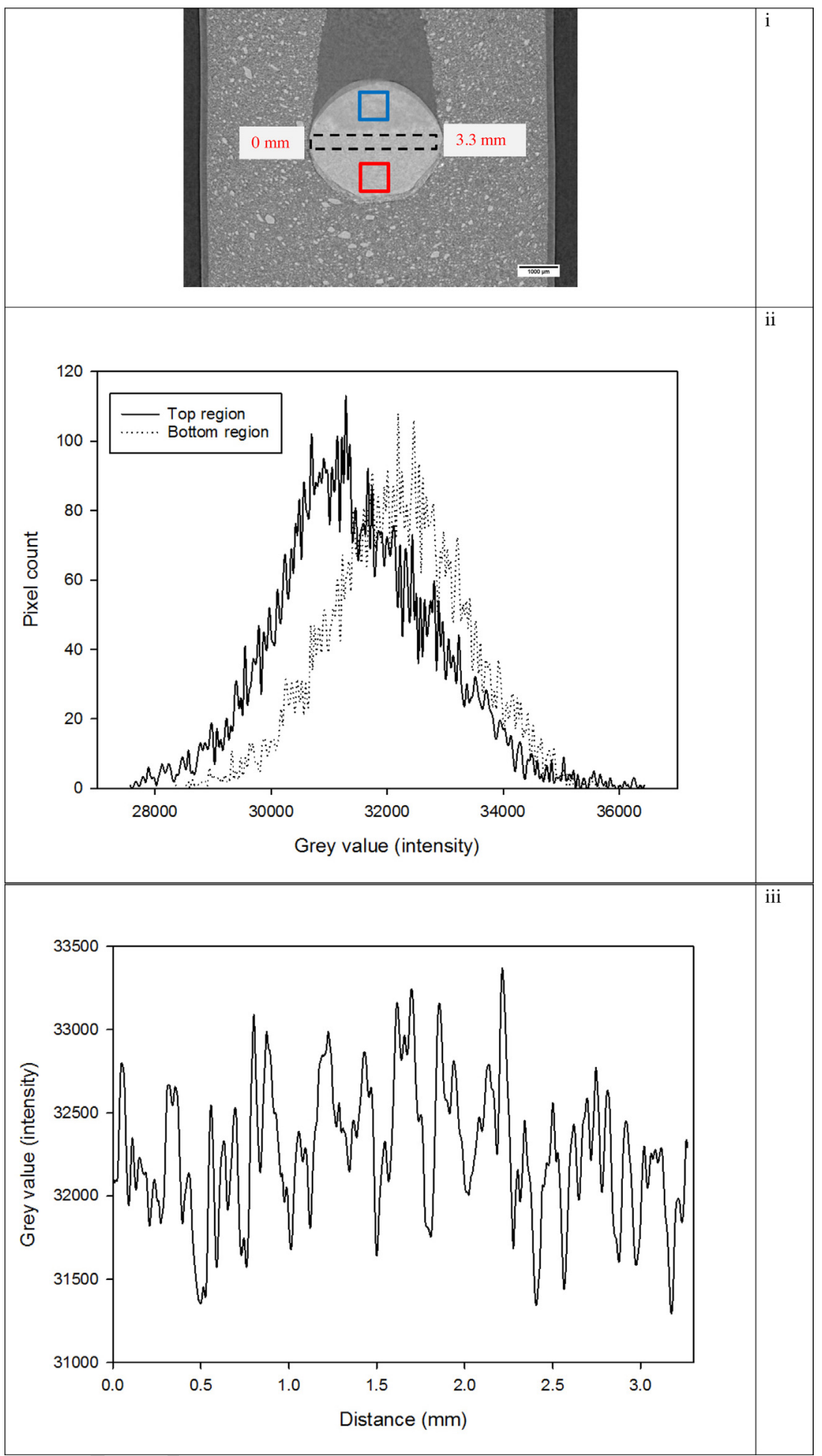

Q12 Fig. 19 - (i) Scan 5A from Fig. 4 for the $5 \mu$ l water droplet after $330 \mathrm{~min}$, (ii) Grey value for top and bottom boxed regions, (iii) Grey value for black dashed line. (For interpretation of the references to colour in the text, the reader is referred to the web version of this article.)

the droplet is coming into contact with sucrose which is moving into the droplet. Therefore, the lower portion of the water droplet will contain more sucrose in comparison to the top portion of the water droplet. The top portion of the droplet will contain a sucrose solution based on the previous sucrose which entered the droplet and has now dissolved, and the lower portion containing solid sucrose which has entered as the droplet moves, thus creating a concentration gradient of sucrose within the droplet.
A 90 pixels $\times 90$ pixels box was drawn within the top region of the droplet as can be seen in Fig. 19(i), using ImageJ to determine the grey scale distribution. A similar box was drawn within the lower region of the droplet. The blue and red boxes in Fig. 19(i) show the measured regions. The distribution of the grey values of these boxes can be seen in Fig. 19(ii) showing that from the top region to the bottom one there is a shift to the right showing the portion is brighter, which as mentioned previously is ascribed to the presence of sucrose (the 


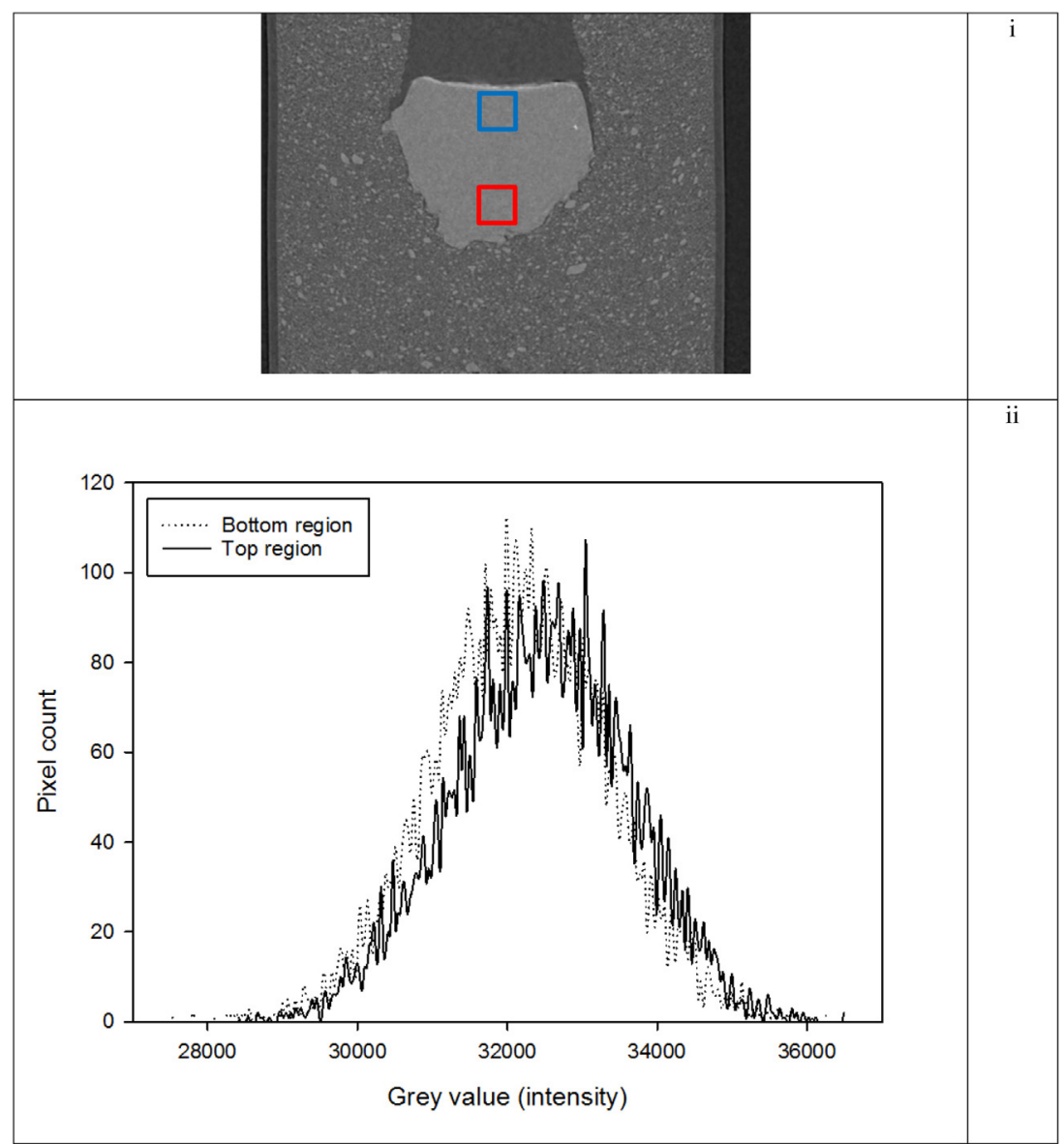

Fig. 20 - (i) Scan 8A from Fig. 4 for the $5 \mu \mathrm{l}$ water droplet after $600 \mathrm{~min}$, (ii) Grey value for top and bottom boxed regions.

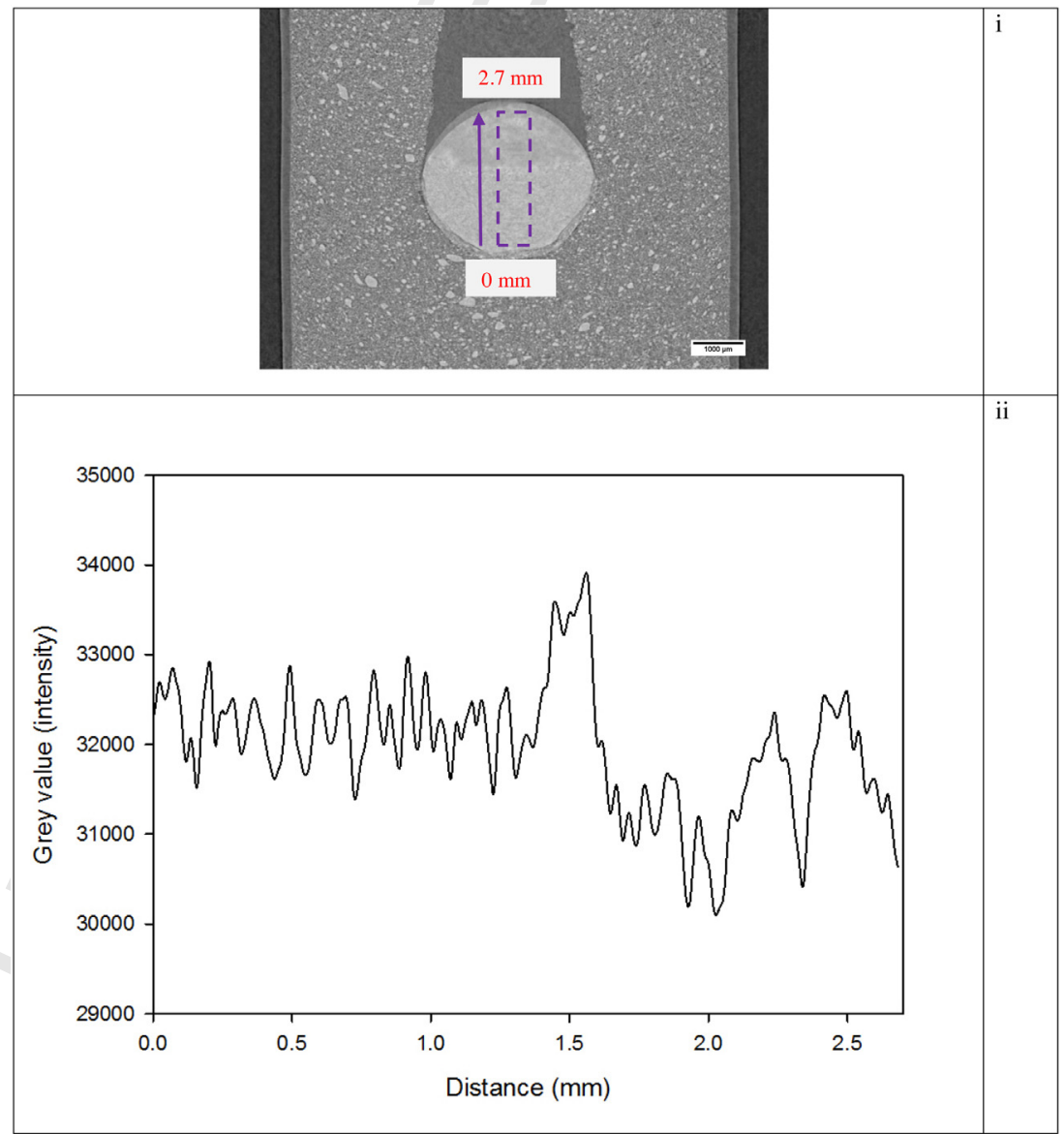

Fig. 21 - (i) Scan 5A from Fig. 4 for the $5 \mu \mathrm{l}$ water droplet after $330 \mathrm{~min}$, (ii) Grey value for the vertical dashed purple box. 


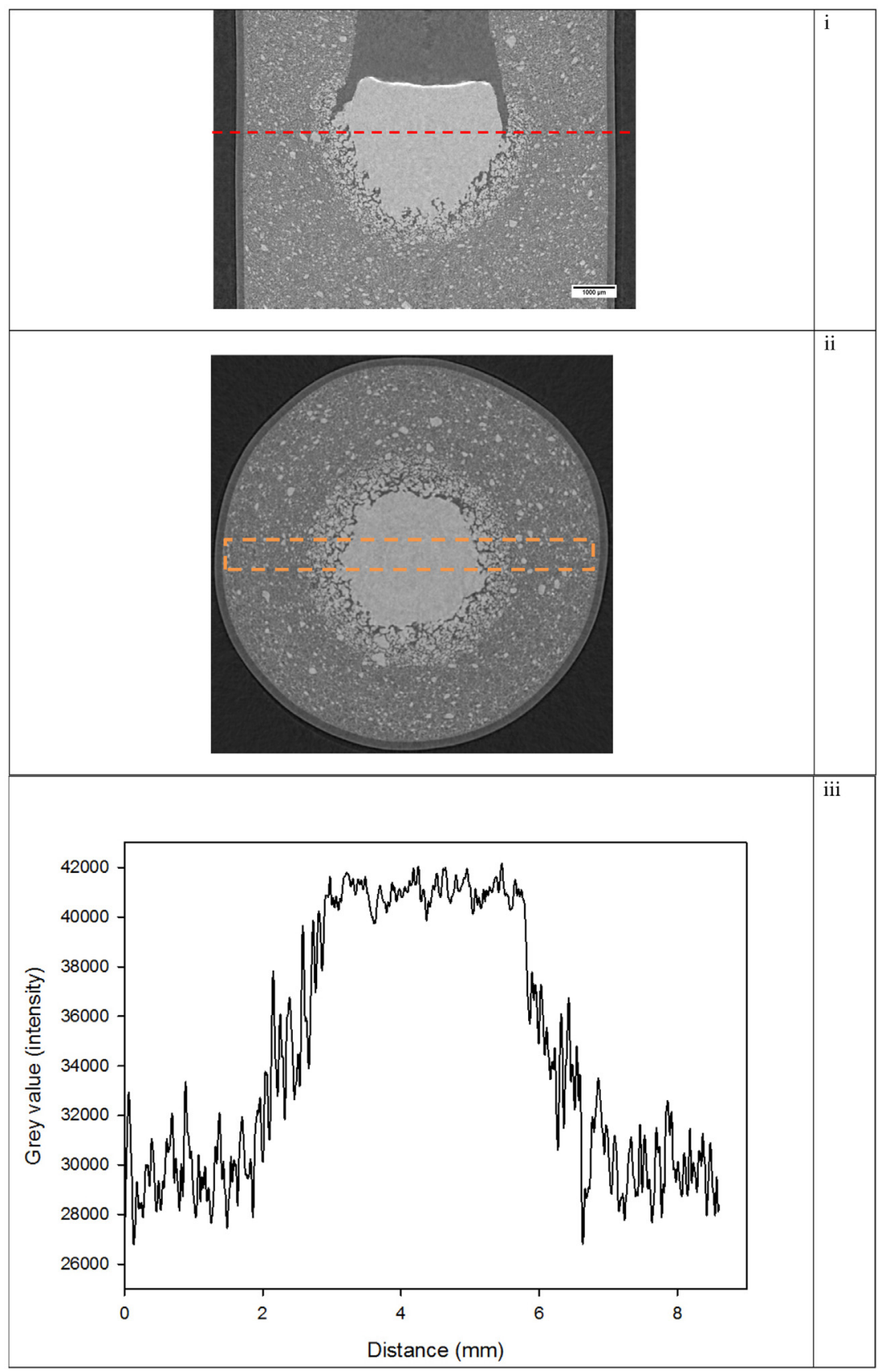

Fig. 22 - (i) Scan 9A from Fig. 4 for the $5 \mu \mathrm{l}$ water droplet after $1080 \mathrm{~min}$, (ii) Axial slice for red line, (iii) Grey value for the vertical dashed orange box. (For interpretation of the references to colour in the text, the reader is referred to the web version of this article.)

mean grey value of the top region is 31,500 , whereas for the bottom region it is 32,200 ).

The grey profile of the horizontal black dashed box in Fig. 19(i) can be seen in Fig. 19(iii). In this case, the grey value of the suspension of oil and sugar is lower than the water droplet with sucrose. The grey profile of the droplet is fairly consistent in the range of 31,500-33,000. The diameter of the droplet for Fig. 19(i) is $3.3 \mathrm{~mm}$, which was measured using the ImageJ software. This shows the droplet has grown substantially from scan $1 \mathrm{~A}$ when the droplet diameter was $2.1 \mathrm{~mm}$. This increase in diameter has been ascribed to the large amount of sucrose which has entered the droplet.

Comparable boxes were drawn on scan $5 \mathrm{~B}$ in Fig. 5 for the $10 \mu$ l water droplet, which showed an analogous trend. By scans $8 \mathrm{~A}$ and $8 \mathrm{~B}$, the droplet grey value was relatively uniform throughout the droplet, as significant sucrose had entered the droplet to be present throughout the whole droplet. Similar boxes were drawn for scan 8A in Fig. 20(i) which can be seen in Fig. 20(ii) where the top and bottom regions have a similar grey range value with a mean of 32,450 and 32,200 , respectively. This shows even though the mean grey value is changing for the whole droplet (as shown in Fig. 18 for the $5 \mu l$ and $10 \mu l$ water droplets, respectively), within the droplet itself there is a grey scale range, corresponding to the presence of sucrose as it enters into the droplet. This shows X-ray CT can successfully be employed to track the movement of sucrose into the droplet as well as the movement of sucrose within the droplet.

For Fig. 21(i), this time the grey value profile from the lower region of the droplet to the upper region of the droplet was measured (vertical dashed purple box, from the bottom to the top) which can be seen in Fig. 21(ii). It can be seen for the lower portion (from 0 to $1.5 \mathrm{~mm}$ ) the grey value is fairly consistent 
at approximately 32,000 . At the region of $1.5 \mathrm{~mm}$, it drops to 31,000 , which is believed to be the location change within the water droplet between the presence of solid sucrose below this region and the absence of solid sucrose above this region.

An enlarged image of scan 9A from Fig. 4, taken after 1080 min, can be seen in Fig. 22(i). As mentioned, after the droplet movement into the suspension slows down as the capacity to take any further sucrose from the bulk suspension is not possible, leads to a phase of water now moving out as shown schematically in Fig. 11 as stage 5, which is believed to be responsible for the further axial spreading of water. An axial slice along the Y-axis (in relation to Fig. 2) for the dashed red line shown in Fig. 22(i) can be seen in Fig. 22(ii). At the centre of the image, the droplet of water and sucrose can be seen in the centre with a layer of oil around it, then some further spreading axially of the water into the surrounding suspension. Larger sucrose crystals within the suspension can be seen, but around the bulk water in the centre, an outer circle can be seen which shows how far the water has spread. There also seems to be a consolidation of sucrose particles which are attracted towards water, due to their hydrophilic nature.

A plot of the change in the grey value for the vertical dashed region (orange rectangle) in Fig. 22(ii) can be seen in Fig. 22(iii). It can be seen how the region of sucrose and sunflower oil (between 0-2 mm) has a consistent greyscale value and as you approach the centre of the image (between $2-3 \mathrm{~mm}$ ), there is a sudden sharp increase in the grey value to where the sucrose and water is located (between $3-6 \mathrm{~mm}$ ), and then a sharp decrease back down (between $6-7 \mathrm{~mm}$ ) as the area of sucrose and sunflower oil on the right of the image (between $7-9 \mathrm{~mm}$ ) is approached. This demonstrates clear changes in the transformation between the different regions, which can be tracked quantitatively due to the high resolution offered by the scans conducted using synchrotron radiation.

\section{Conclusion}

In this research the fate of a secondary immiscible liquid droplet (water or saturated sucrose solution) when added to a suspension of crystalline sucrose in a continuous phase of sunflower oil was investigated. The progression of the different droplets was monitored using synchrotron radiation computed microtomography as a non-invasive approach to track the movement of the droplet in three-dimensions within the volume of the suspension through a time lapse of scans. Changes in grey value of the droplet allowed the movement of sucrose into the aqueous liquid phase to be followed, and changes to droplet shape and the suspension in its vicinity offer insight into the range of mechanisms that might redistribute the liquid during short and long term storage.

The method of X-ray CT utilised in this paper highlights the potential of using this approach to study the storage of products which contain several domains with different water activities. It shows the possibility of tracking the movement taking place, which is important to understanding the shelflife of products.

\section{Acknowledgements}

9 We would like to acknowledge the financial support of BBSRC (Grant no. BB/K501542/1) in supporting this research and the staff of the X-ray imaging beamline SYRMEP for the support during beamtime (Proposal: 20150246). Networking support was provided by the EXTREMA COST Action MP1207.

\section{References}

Asadi, M., 2005. Tables. In: Beet-Sugar Handbook. John Wiley \& Sons, Inc, pp. 779-801.

Baruchel, J., et al., 2000. X-Ray Tomography in Material Science, General Principles. Hermes Science Publications, Paris.

Brun, F., et al., 2015. Enhanced and flexible software tools for $\mathrm{X}$-ray tomography experiments at the Italian synchrotron radiation facility Elettra. Fundam. Inform. 141 (2-3), 233-243.

Bucke, C., 1995. Sucrose, Properties and Applications, Vol. 69. Chapman \& Hall, London.

Chevallier, S., et al., 2014. Determining the cellular structure of two cereal food foams by X-ray micro-tomography. Food Biophys. 9 (3), 219-228.

Cloetens, P., et al., 1996. Phase objects in synchrotron radiation hard X-ray imaging. J. Phys. D: Appl. Phys. 29 (1), 133.

Falcone, P.M., et al., 2004. A novel approach to the study of bread porous structure: phase-contrast X-ray microtomography. J. Food Sci. 69 (1), FEP38-FEP43.

Falcone, P.M., et al., 2006. Imaging Techniques for the Study of Food Microstructure: A Review, in Advances in Food and Nutrition Research. Academic Press, pp. 205-263.

Frisullo, P., Conte, A., Del Nobile, M.A., 2010a. A novel approach to study biscuits and breadsticks using X-ray computed tomography. J. Food Sci. 75 (6), E353-E358.

Frisullo, P., et al., 2010b. Microstructural characterization of multiphase chocolate using X-ray microtomography. J. Food Sci. 75 (7), E469-E476.

Ghosh, V., et al., 2004. Diffusion of moisture through chocolate-flavoured confectionery coatings. Food Bioprod. Process. 82 (1), 35-43.

Herremans, E., et al., 2014. X-ray CT for quantitative food microstructure engineering: the apple case. Nucl. Instrum. Methods Phys. Res. Sect. B: Beam Interact. Mater. Atoms 324 (0), 88-94

HirschmÜLler, H., 2013. Chapter 2-physical properties of sucrose A2-Honig, Pieter. In: Principles of Sugar Technology. Elsevier, S-Hertogenbosch, pp. 18-74.

Islam, S.F., et al., 2016. Movement of a secondary immiscible liquid in a suspension using a non-invasive technique. Chem. Eng. Res. Des. 110, 160-170.

Johansson, D., Bergenståhl, B., 1992. The influence of food emulsifiers on fat and sugar dispersions in oils. I. Adsorption, sedimentation. J. Am. Oil Chem. Soc. 69 (8), 705-717.

Kak, C.A., Slaney, M., 1988. Principles of Computerized Tomographic Imaging. IEEE Press, New York.

Killian, L., Coupland, J., 2012. Manufacture and application of water-in-oil emulsions to induce the aggregation of sucrose crystals in oil: a model for melt-resistant chocolate. Food Biophys. 7 (2), 124-131.

Koos, E., Willenbacher, N., 2011. Capillary forces in suspension rheology. Science 331 (6019), 897-900.

Koos, E., Willenbacher, N., 2012. Particle configurations and gelation in capillary suspensions. Soft Matter 8 (14), 3988

Koos, E., et al., 2012. Tuning suspension rheology using capillary forces. Soft Matter 8 (24), 6620.

Laverse, J., et al., 2012. X-ray microtomography to study the microstructure of mayonnaise. J. Food Eng. 108 (1), 225-231.

Mewis, J., Wagner, N.J., 2012. Colloidal Suspension Rheology. Cambridge University Press, Cambridge.

Myers, G.R., et al., 2007. Polychromatic cone-beam phase-contrast tomography. Phys. Rev. A 76 (4), 045804.

Negreiros, A.A., et al., 2015. Microscale study of particle agglomeration in oil-based food suspensions: the effect of binding liquid. Powder Technol. 270 (Part B(0)), 528-536.

Paganin, D., et al., 2002. Simultaneous phase and amplitude extraction from a single defocused image of a homogeneous object. J. Microsc. 206 (1), 33-40.

Pittia, P., et al., 2011. Evaluation of microstructural properties of coffee beans by synchrotron X-ray microtomography: a methodological approach. J. Food Sci. 76 (2), E222-E231. 
9210 Reinke, S.K., et al., 2015. Synchrotron X-ray microtomography reveals interior microstructure of multicomponent food 22@11 materials such as chocolate. J. Food Eng.

925 Yuan, Q., Hanselmann, W., Anantheswaran, R.C., 2009.

926 Characterization of vapor-induced and liquid-induced

927 moisture migration through fractionated palm kernel (PKO) based multiphase systems. J. Food Eng. 95 (3), 460-470.
Yuan, Q., Hanselmann, W., Anantheswaran, R.C., 2012. Moisture and sucrose transfer in fractionated palm kernel (PKO) based films immersed in liquid solutions. J. Food Eng. 108 (1), 13-21. 